\title{
CCL17-producing CDC2s are essential in end-stage lupus nephritis and averted by a parasitic infection
}

\author{
Laura Amo, Hemanta K. Kole, Bethany Scott, Chen-Feng Qi, Juan Wu, and Silvia Bolland \\ Laboratory of Immunogenetics, Division of Intramural Research, National Institute of Allergy and Infectious Diseases (NIAID), NIH, Rockville, Maryland, USA
}

\begin{abstract}
Lupus nephritis is a severe organ manifestation in systemic lupus erythematosus leading to kidney failure in a subset of patients. In lupus-prone mice, controlled infection with Plasmodium parasites protects against the progression of autoimmune pathology including lethal glomerulonephritis. Here, we demonstrate that parasite-induced protection was not due to a systemic effect of infection on autoimmunity as previously assumed, but rather to specific alterations in immune cell infiltrates into kidneys and renal draining lymph nodes. Infection of lupus-prone mice with a Plasmodium parasite did not reduce the levels or specificities of autoreactive antibodies, vasculitis, immune complex-induced innate activation, or hypoxia. Instead, infection uniquely reduced kidney-infiltrating CCL17-producing bone marrow-derived type 2 inflammatory dendritic cells (iDC2s). Bone marrow reconstitution experiments revealed that infection with Plasmodium caused alterations in bone marrow cells that hindered the ability of DC2s to infiltrate the kidneys. The essential role for CCL17 in lupus nephritis was confirmed by in vivo depletion with a blocking antibody, which reduced kidney pathology and immune infiltrates, while bypassing the need for parasitic infection. Therefore, infiltration into the kidneys of iDC2s, with the potential to prime local adaptive responses, is an essential regulated event in the transition from manageable glomerulonephritis to lethal tubular injury.
\end{abstract}

\section{Introduction}

Lupus nephritis is one of the most severe organ manifestations of systemic lupus erythematosus (SLE) and a main cause of morbidity in patients who progress to end-stage kidney disease (1). Several factors contribute to the type of renal injury associated with SLE: (a) tissue deposition of immune complexes (ICs) that are formed when high titers of autoantibodies are present in the circulation; (b) local complement fixation, FcR activation, and expression of cytokines induced by the presence of ICs; and (c) leukocyte recruitment to the kidney elicited by chemotactic signals. Although the presence of circulating autoreactive antibodies and consequent IC deposition in the kidneys are common occurrences in SLE, severe kidney pathology is only observed in a subset of patients (2-4). Thus, it is possible that a further step beyond IC-mediated inflammation might be required for the progression of end-stage kidney disease.

Destructive kidney pathology has been correlated with immune cell infiltrates in both human SLE and mouse models (5, 6). In fact, the complexity of the infiltrates has been revealed by single-cell RNA-Seq in human SLE kidney biopsy samples, pointing to a type I IFN expression profile and fibrotic pathways in distinct types of nephritis (7). Recent studies have also implicated hypoxia and metabolic stress in the transition to more severe nephritis (8-10) by recruiting T cells to the injured site (11). How-

Conflict of interest: The authors have declared that no conflict of interest exists. Copyright: (5) 2021, American Society for Clinical Investigation.

Submitted: January 22, 2021; Accepted: April 21, 2021; Published: June 1, 2021.

Reference information: J Clin Invest. 2021;131(11):e148000.

https://doi.org/10.1172/JCI148000. ever, there are still unclear aspects regarding the sequential steps and the role of these molecules in the most destructive phase of nephritis. In this regard, immune cell infiltrates in tubular areas have been reported to form tertiary lymphoid structures that contribute to lymphocyte priming within the kidneys (12). This priming might require the presence of activated dendritic cells (DCs) able to initiate adaptive responses involving kidney-specific antigens. Studies using the MRL-lpr mouse model found that DCs were not required for systemic lymphocyte activation, but they promoted tissue damage (13). The particular type of DC, the origin of these cells, or the way they might be recruited to the kidneys are still open inquiries. Studies using NZB/W mice revealed that infiltration of both activated macrophages and DCs was associated with proteinuria onset and that both active immune recruitment and local innate mechanisms were implicated $(8,14-16)$. Analysis of kidney infiltrates in a transgenic model of induced nephritis indicated a role for kidney DC activation in the progression of renal disease $(16,17)$. In these previous studies, DCs were defined by their expression of CD11c and high expression of MHC-II. However, recent work that fully characterizes DCs in inflammatory conditions points to the need for more defining markers (CD26, CD88) to distinguish activated DCs from inflammation-associated macrophages (18). Infiltrating inflammatory DCs have been clearly characterized in lungs after various infections, but they have been more challenging to define in other organs such as the kidneys (19).

To investigate mechanisms underlying lupus nephritis, we considered previous reports of a life-long protective effect of Plasmodium infection that completely reduced the incidence of lethal lupus nephritis in NZB/W mice (20). For the past several decades, 
A

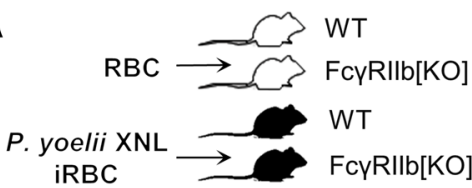

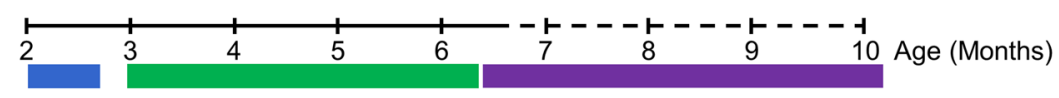

Py infection SLE symptoms analysis
SLE survival analysis

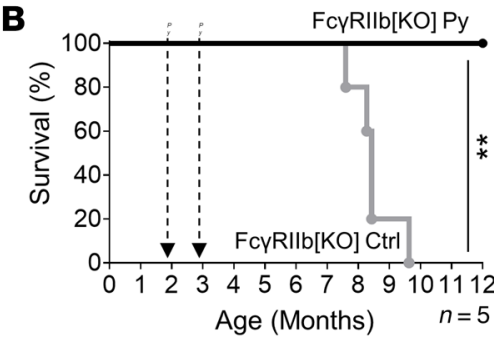

C
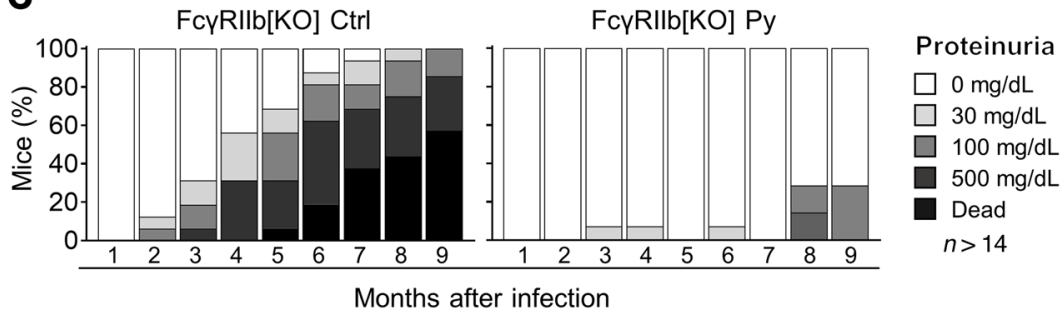

E
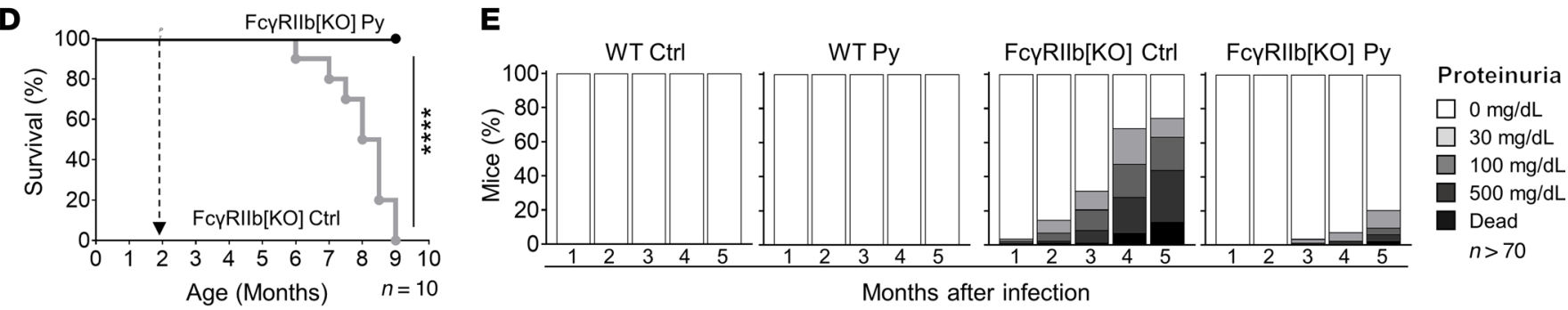

$\mathbf{F}$
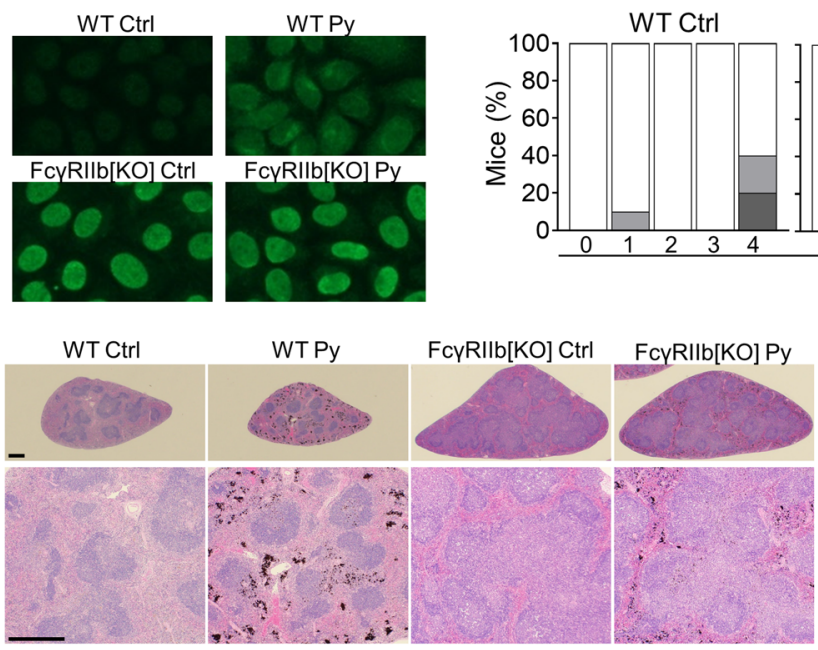

WT Py

FcyRIllb[KO] Ctrl

FcyRllb[KO] Py

ANA

$\square 0$

$\square$ 1:100

$\square$ 1:300

$\square$ 1:900

$\square$ 1:2700

$1: 8100$

$n>10$

G
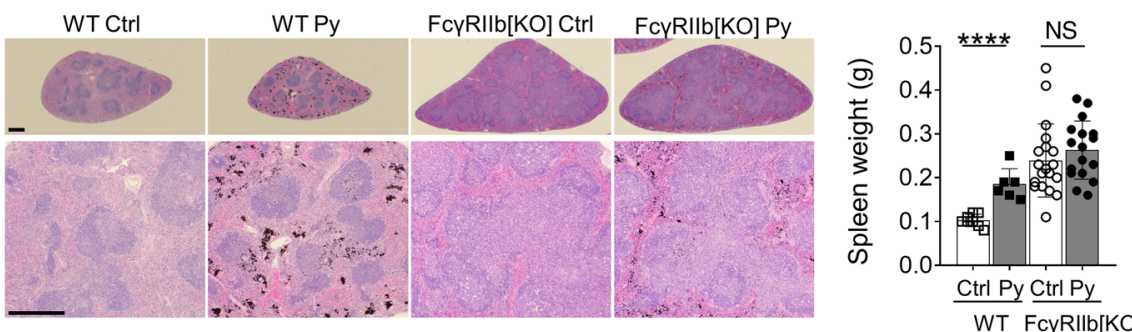

WT FcyRIIb[KO]

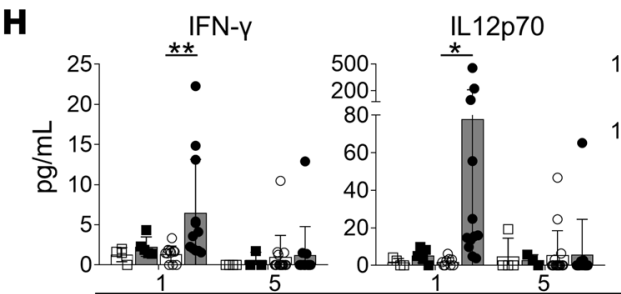

MCP-1

IL-10
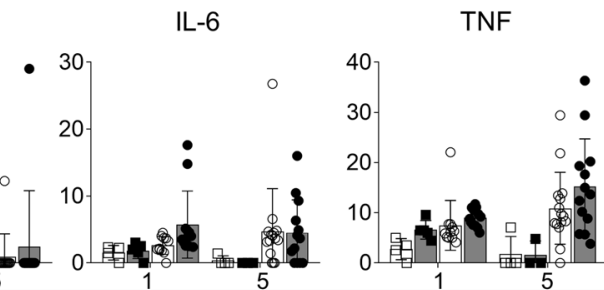

Months after infection

Figure 1. Parasite infection induces autoreactivity while reducing kidney pathology. (A) Schematic representation of the experimental design and timeframes applicable throughout the study (further details are presented in Supplemental Figure 1, A and B). (B-E) Cumulative survival curve and percentage

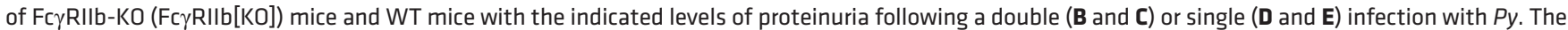
time point of $P y$ infection is indicated by arrows ( $n=5$ and $n=10$ in the survival analysis). Proteinuria levels were measured by chemical strips and are shown in grayscale. (C) $n=14$ (months 1-6), $n=9$ (months 7-8), and $n=7$ (month 9). (E) $n=7$ and $n=15-22$ for the WT and Fc $\gamma$ RIllb-KO groups, respectively. (F) ANA detection in serum of WT or FcyRllb-KO mice infected with Py at 2 months of age. Shown are representative images of the immunofluorescence assay (original magnification, $\times 20$ ) and graphs indicating the percentage of mice with the indicated ANA levels. ANA positivity in different serum dilutions is shown in grayscale. $n=7$ in WT and $n=22$ for the Fc $\gamma$ RIIb-KO groups (months $0-2$ ), and $n=5$ for the WT and $n=14$ for the Fc $\gamma R$ RIIb-KO groups (months 3-4). (G) Representative H\&E-stained images (scale bars: $1 \mathrm{~mm}$ [top] and $500 \mu \mathrm{m}$ [bottom]) and spleen weights for WT ( $n=6)$ and Fc $\gamma \mathrm{RIIb}-\mathrm{KO}(n=17)$ mice $5 \mathrm{months}$ after a single infection with $P y$. (H) Serum cytokine levels (IFN- $\gamma$, IL-12p70, MCP-1, IL-10, IL-6, and TNF) measured 1 and 5 months after a single $P y$ infection. Genotype and infection status are indicated in G. $n=4$ and $n=10$ for the WT and Fc $\gamma R$ Rllb-KO groups. Data are presented as the mean \pm SD. ${ }^{*} P<0.05$, ${ }^{* *} P<$ 0.01 , and ${ }^{* * *} P<0.0001$, by log-rank (Mantel-Cox) test was used for survival comparisons (B and $\mathbf{D}$ ) and 1- and 2-way ANOVA (G and $\left.\mathbf{H}\right)$. Ctrl, control. 
there has been little effort to understand how this protective effect of malaria alters the autoimmune phenotype in SLE. Past investigations pointed to a systemic reduction of the autoimmune response and inflammatory mediators and a putative reduction of macrophage function following a parasitic infection (21). The data presented here revealed a more restricted protective effect of infection with Plasmodium that did not reduce systemic autoimmunity or inflammation while preventing end-stage lethal nephritis. For these experiments, we infected the well-characterized SLE mouse model Fc $\gamma$ RIIb-KO (22) with the nonlethal strain of malaria Plasmodium yoelii 17XNL-GFP (Py), which achieves a blood-stage, self-resolving infection without the use of any antimalarial treatment (23). The results revealed that infection with Plasmodium induced bone marrow (BM) alterations that specifically targeted kidney-infiltrating DCs. Analysis of the differential effects on local chemokine expression uncovered a potential role for a small number of CCL17-producing inflammatory DCs in the transition to end-stage kidney disease. Overall, the results obtained in this work reveal a possible mechanism underlying the severe pathology of lupus nephritis, identifying cells and a chemokine with an essential role in disease progression.

\section{Results}

Parasite infection promotes autoreactivity but protects against lethal kidney pathology. To explore mechanistic aspects underlying a potential protective effect of controlled infection with a malaria parasite in the progression of autoimmune pathology, we infected lupus-prone Fc $\gamma$ RIIb-KO mice with $P y$ at a presymptomatic age and followed them for signs of disease for up to 12 months (Figure 1A and Supplemental Figure 1, A and B; supplemental material available online with this article; https://doi.org/10.1172/ JCI148000DS1). Fc $\gamma$ RIIb-KO mice develop spontaneous lupuslike disease characterized by chronic systemic immune activation, high serum autoantibody titers, widespread vasculitis, and lethal glomerulonephritis (22). All Fc $\gamma$ RIIb-KO mice inoculated with $P y$-infected red blood cells (iRBCs), either in single or repeated injections, survived past 9 months of age, a time point at which all uninfected control mice had succumbed to autoimmune disease (Figure 1, B and D). The increased survival rate of parasite-infected Fc $\gamma$ RIIb-KO mice correlated with a highly significant improvement in kidney function measured by proteinuria. Less than $20 \%$ of the mice had measurable levels of proteinuria by 7 months of age when inoculated with a single dose of $P y$ (Figure 1E and Supplemental Figure 1D), or by 10-11 months of age after double inoculation (Figure 1C and Supplemental Figure 1C), in contrast to the complete penetrance of proteinuria at that age observed in the uninfected Fc $\gamma$ RIIb-KO controls. The increased rate of survival after $P y$ infection did not correlate with a reduction in autoantibody titers: infected Fc $\gamma$ RIIb-KO mice had serum antinuclear autoantibodies (ANAs) with equal or even higher (1 month after infection) titers than did the uninfected Fc $\gamma$ RIIb-KO controls (Figure $1 \mathrm{~F}$ and Supplemental Figure 1E). Infection of Fc $\gamma$ RIIb-KO mice with $P y$ did not alter other measures of systemic autoimmunity, such as splenomegaly, or the presence of spontaneous germinal centers typical of this strain (Figure 1G). Inflammatory cytokines were elevated soon after $P y$ infection, however, 5 months later they were at the same levels as those in the control animals (Figure
1H). Overall, these results suggest that $P y$ infection of Fc $\gamma$ RIIb-KO mice protects from lethal kidney disease, without reducing the typical features of systemic autoimmunity.

Parasite infection does not alter the autoreactive specificity profile or IC deposition in kidneys of lupus-prone mice. We found that infection with $P y$ increased overall titers of serum ANAs in both WT mice and FcrRIIb-KO mice (Figure 1F). It is also possible that malaria infection changed certain autoantibody specificities characteristic of lupus-prone mice in a way that would make them less pathogenic or even protective. To test this possibility, we performed an IgG autoantigen microarray with 128 self-antigens selected from those commonly targeted in a variety of autoimmune diseases (AutoAg microarray super panel, Genomics Core Facility, UT Southwestern, Dallas, Texas, USA) (24). We determined that serum IgGs from malaria-infected Fc $\gamma$ RIIb-KO mice had the same pattern of antigen specificities as serum IgG from uninfected $\mathrm{Fc} \gamma$ RIIb-KO mice. Some autoreactive specificities ( $\beta 2$-glycoprotein I, C9, CRP, Jo-1, nucleosome Ag, PL-7, ssDNA) even increased titers with the infection (Figure 2A). We obtained similar results when testing IgM autoreactive specificities (Supplemental Figure 2A). Total serum IgM, or IgG of any isotype, showed equal titers in $\mathrm{Fc} \gamma$ RIIb-KO mice, whether infected with $P y$ or uninfected (Figure 2B and Supplemental Figure 2B). Next, we tested whether autoantibodies induced by Plasmodium infection might have a protective effect in passive transfer experiments, as had been previously described using serum from infected BALB/c mice (25). We did not observe a significant reduction in proteinuria in mice injected with serum from malaria-infected Fc $\gamma$ RIIb-KO donors compared with those injected with serum from uninfected donors (Figure 2C). We then measured glomerular IC deposition in the kidneys of Fc $\gamma$ RIIb-KO mice 5 months after infection. Neither complement C3 nor various IgG isotypes showed differences in the amount of kidney deposits in infected mice compared with controls (Figure 2D and Supplemental Figure 2C). These results showed that infection with a malaria parasite conferred protection from kidney disease at a stage beyond IC deposition and complement fixation.

Parasite infection of lupus-prone mice prevents severe kidney damage but not glomerular inflammation. Fc $\gamma \mathrm{RIIb}-\mathrm{KO}$ mice develop an autoimmune kidney pathology that progresses in severity: it starts with glomerular inflammation with mild diffuse mesangial hypercellularity, then evolves to proliferative glomerulonephritis with sclerotic lesions, and ends in a final destructive phase that involves fibrosis and leukocyte infiltration (Supplemental Figure 3A). Histopathological examination of kidneys from uninfected 7-month-old Fc $\gamma$ RIIb-KO mice showed extensive glomerular destruction, interstitial cell infiltration, protein deposits, and vasculitis (Figure 3A, H\&E). In contrast, kidneys from $P y$-infected Fc $\gamma$ RIIb-KO mice showed enlarged glomeruli compared with those of WT control mice, but very minimal interstitial infiltration and glomerular destruction. Using periodic acid silver methenamine stain (PAMS), we determined that glomeruli in Fc $\gamma$ RIIb-KO kidneys presented obvious basement membrane abnormalities, with Bowman's capsule rupture and sclerotic features. Glomeruli from malaria-infected FcyRIIb-KO kidneys had intact basement membranes (Figure 3A, PAMS). Malaria parasite infection also resulted in a substantial reduction in cellular apoptosis in the kidneys compared with uninfected controls (Figure 3A, TUNEL). Trans- 
A
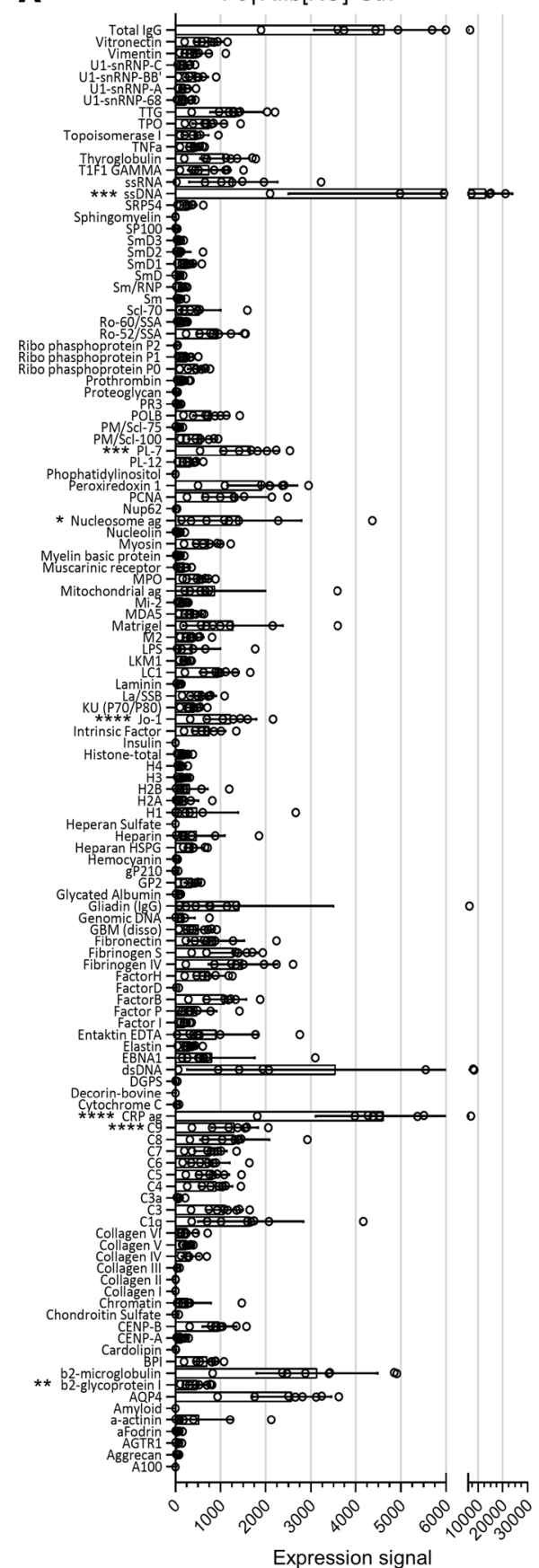

FcyRllb[KO] Py

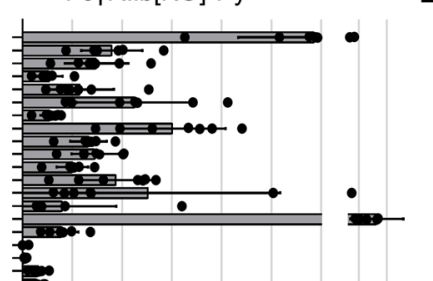

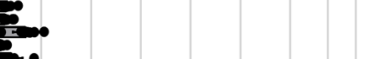
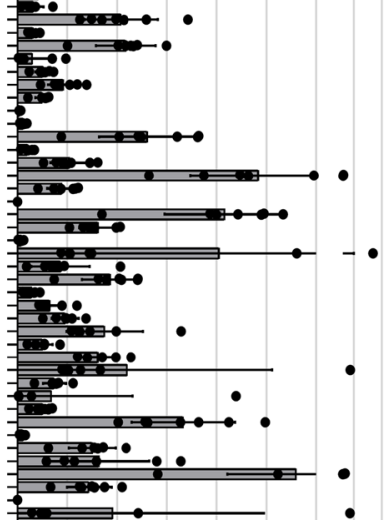

D
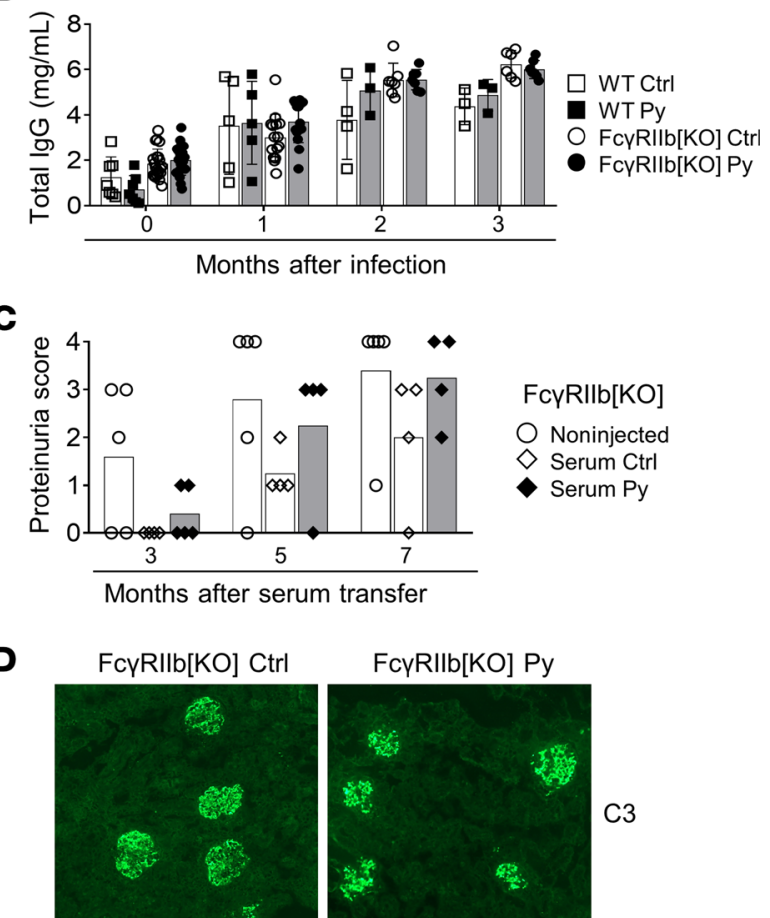

Months after infection

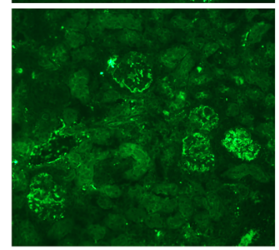

FcyRIlb[KO] Py
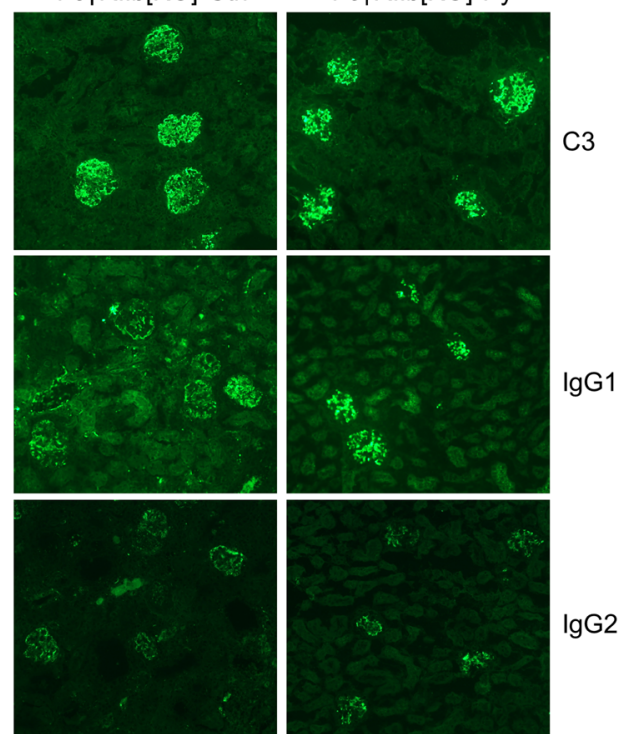

$\operatorname{lgG} 2 \mathrm{a} / \mathrm{c}$
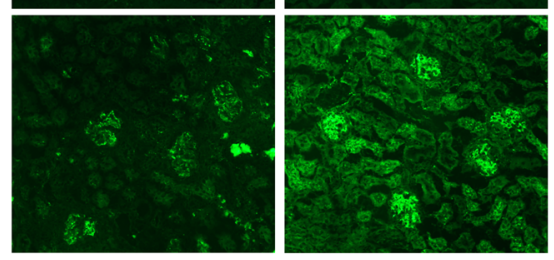

$\operatorname{lgG} 2 \mathrm{~b}$

Figure 2. Parasite infection does not alter the autoreactive specificity profile or IC deposition in FcyRIIb-KO mice. (A) Quantification of the IgG autoantibody

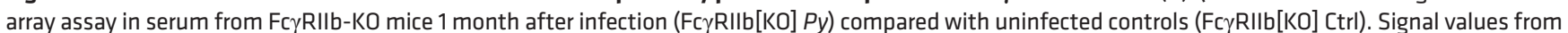
the Autoantigen Microarray Super Panel (24) are shown. $n=8$ (control mice) and $n=7$ (Py-infected mice). (B) Total serum IgG quantification by ELISA for WT mice and FcyRIlb-KO mice after Py infection at the indicated time points. $n=7,5,4$, and 3 for the WT groups, and $n=22,15,7$, and 7 for the Fc $\gamma$ RIIb-KO groups for months $0,1,2$, and 3, respectively. (C) Proteinuria score for Fc $\gamma R$ IIlb-KO mice who received i.v injections (3 injections, once per week) of serum from Fc $\gamma$ RIIb-KO control or Py-infected mice. The proteinuria scores were defined as follows: 1 ( $30 \mathrm{mg} / \mathrm{dL}), 2$ (100 mg/dL), 3 ( $500 \mathrm{mg} / \mathrm{dL}$ ), and 4 (death due to kidney failure). $n=5$, noninjected control mice and $n=4$, serum-injected groups. (D) FcyRIlb-KO control and Py-infected kidney sections stained with anti-complement $\mathrm{C} 3$ antibody or anti-IgC1, -IgG2a/c, or -lgG2b antibodies conjugated with FITC $(n=3)$. Data are presented as the mean \pm SD. Group comparisons were made using 2-way ANOVA. ${ }^{*} P<0.05,{ }^{* *} P<0.01,{ }^{* *} P<0.001$, and ${ }^{* * * *} P<0.0001$.

mission electron microscopy (TEM) images showed that infected Fc $\gamma$ RIIb-KO kidneys had intact podocytes with normal foot processes, whereas kidneys from uninfected Fc $\gamma$ RIIb-KO mice had lost the podocyte structure essential for urine filtration (Figure
3A, TEM). Image quantification of all these histological measures indicated that there were no significant differences in glomerular size or Bowman's space areas between infected and uninfected FcyRIIb-KO kidneys, but the kidneys differed significantly in the 

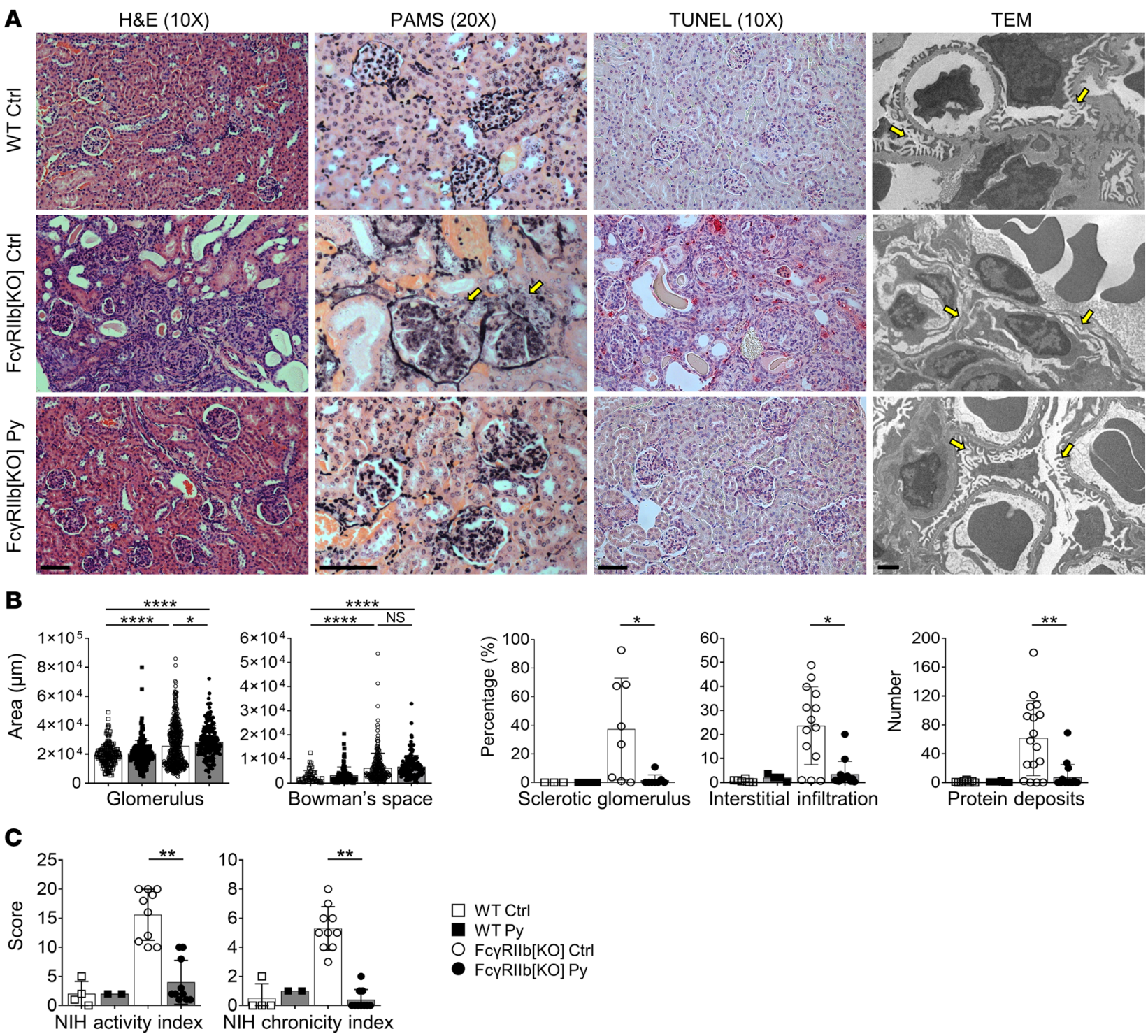

Figure 3. Parasite infection prevents severe kidney injury but does not reduce glomerular inflammation in Fc $\gamma$ RIIb-KO mice. Study of kidney pathology in WT and FcyRIIb-KO mice 5 months after infection with Py. (A) Representative images of kidney sections stained with H\&E $(n=6)$, PAMS $(n=4)$, and TUNEL $(n=2)$ (scale bars: $100 \mu \mathrm{m})$ and TEM images $(n=5)$ (scale bar: $1 \mu \mathrm{m})$. Yellow arrows in the PAMS images indicate noticeable basement membrane abnormalities. Yellow arrows in the TEM images indicate intact (WT control and Fc $\gamma R$ IIlb-KO Py) and destroyed (Fc $\gamma R$ RIIB-KO control) podocyte foot processes. (B) Image) measurements of glomerular and Bowman's space areas (each dot represents 1 glomerulus; $n=2$ WT and $n=5$ Fc $\gamma$ RIlb-KO mice per group), percentage of sclerotic glomeruli, interstitial infiltration, and protein deposit areas in the kidneys (each dot represents 1 mouse). $n=3$ and $n=14$ (sclerotic glomeruli); $n=6$ and $n=14$ (interstitial infiltration) for the WT and Fc $\gamma$ RIIb-KO groups, respectively. (C) NIH activity and chronicity indexes calculated as described in Supplemental Figure 3B. $n=4,2,10$, and 10 for WT control, WT Py, Fc $\gamma R$ Rllb-KO control and Fc $\gamma R$ Illb-KO Py, respectively. Data are presented as the mean \pm SD. Fc $\gamma$ RIIb-KO comparisons were made using the Kruskal-Wallis test. ${ }^{*} P<0.05,{ }^{* *} P<0.01$, and ${ }^{* * *} P<0.0001$.

percentage of sclerotic glomeruli, protein deposits, and interstitial infiltration (Figure 3B). Pathological quantification of kidney injury using the NIH activity and chronicity indexes (26) confirmed that $P y$ infection significantly reduced both the activity and chronicity of nephritis in FcrRIIb-KO mice to levels comparable to those seen in WT mice (Figure 3C and Supplemental Figure 3B).

Parasite infection reduces leukocyte infiltration into kidney, but not liver, of lupus-prone mice. Immunohistological analysis of control Fc $\gamma$ RIIb-KO kidneys showed that infiltrating T cells $\left(\mathrm{CD}^{+}\right)$were located in the cortex, glomerular, and perivascular areas. Myeloid cells $\left(\mathrm{F} 4 / 80^{+}, \mathrm{IBA} 1 / \mathrm{Arg}-1^{+}\right)$had a broad distribution throughout the medulla and cortex with periglomerular extension. In contrast, parasite-infected Fc $\gamma$ RIIb-KO kidneys had minimal infiltration of leukocytes in glomeruli or perivascular areas compared with the lupus control mice (Figure 4A). B cells and neutrophils were found in very low numbers in glomerular and interstitial areas, regardless of infection status (Supplemental Figure 3C).

We performed flow cytometry to quantify $\mathrm{CD} 45^{+}$cells infiltrating the kidneys of Fc $\gamma$ RIIb-KO mice 5 months after infection with $P y$. To discriminate circulating from tissue-infiltrating leukocytes, we injected $3 \mu \mathrm{g}$ anti-mouse CD 45 antibody i.v. 3 minutes before euthanizing the mice (Supplemental Figure 3D). Flow 
A
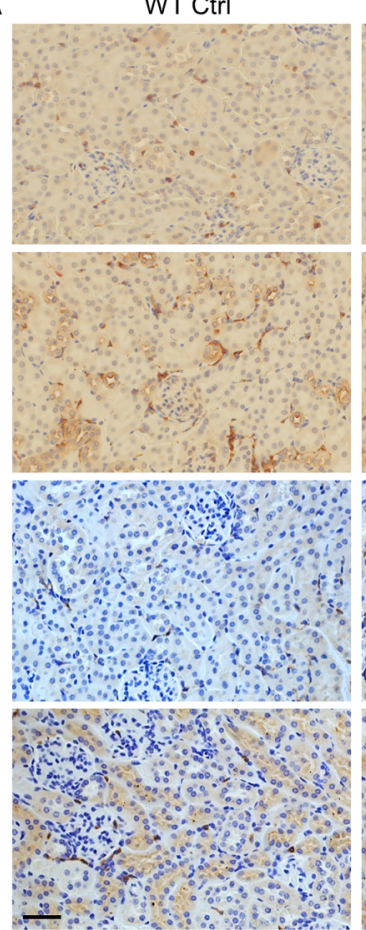

WT Py
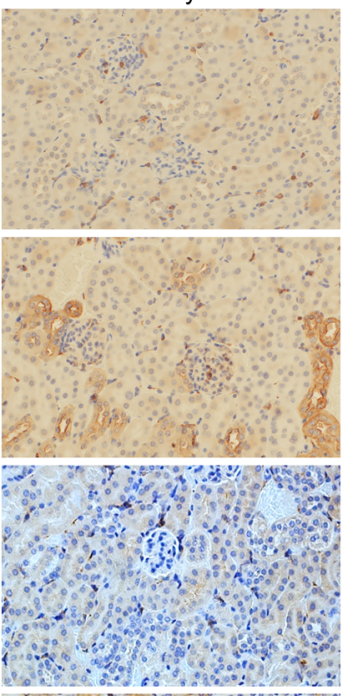

FcyRIlb[KO] Ctrl
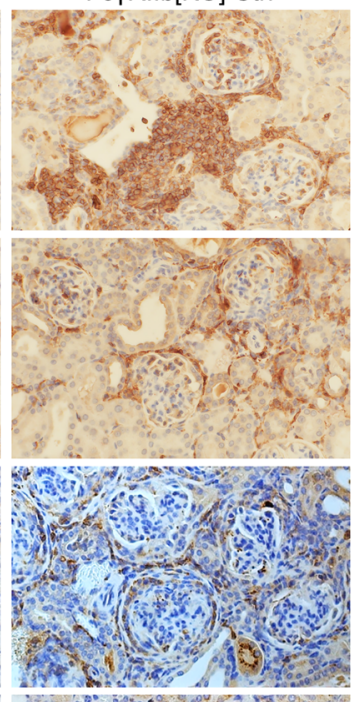

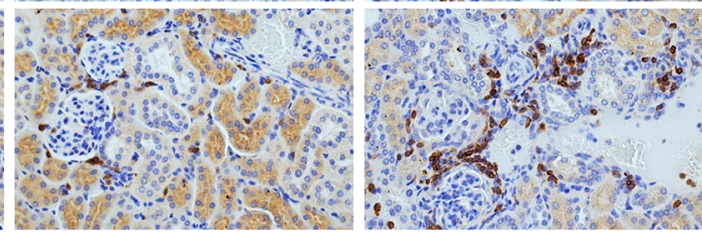

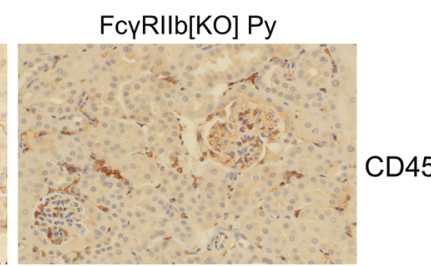
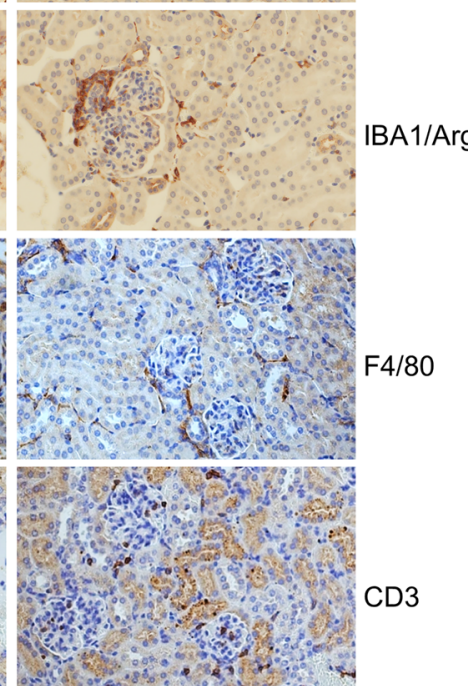

B

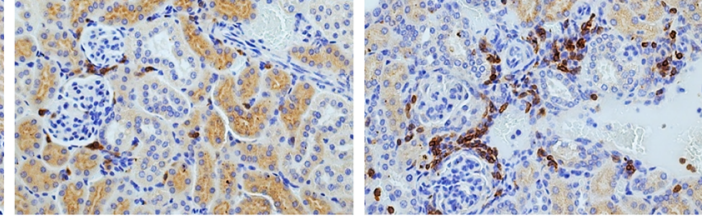

C

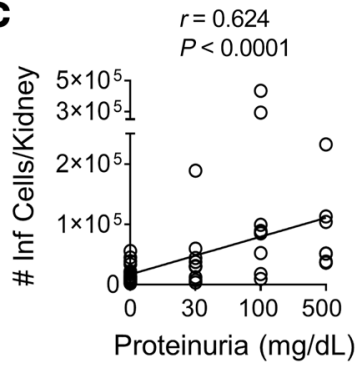

Figure 4. Parasite infection impairs T cell and monocyte infiltration in kidney. Study of kidney-infiltrating cells in WT and FcyRllb-KO mice 5 months after infection with Py. (A) Kidney sections were stained with anti-mouse CD45, -IBA1/Arg1, -F4/80, or -CD3 antibodies (scale bar: $200 \mu \mathrm{m}$ ). (B) Number of infiltrating (Inf) leukocytes (CD45+), myeloid cells (CD11 $\left.\mathrm{b}^{+}\right)$, macrophages (CD11 $\left.\mathrm{b}^{+} \mathrm{F} 4 / 8 \mathrm{O}^{+}\right)$, and $\mathrm{CD} 4^{+} / \mathrm{CD} 8^{+} \mathrm{T}$ cells $\left(\mathrm{TCRb}^{+}\right)$measured by flow cytometry and gated as described in Supplemental Figure 3D and Supplemental Figure 4A. $n=6,6,13$, and 12 for WT control, WT Py-infected, Fc $\gamma R$ Rllb-KO control, and Fc $\gamma R$ Rllb-KO Py-infected mice, respectively. Data are presented as the mean \pm SD. Group comparisons were made using 1-way ANOVA. ${ }^{*} P<0.05$ and ${ }^{* *} P<0.01$. (C) Correlation chart showing the number of kidney-infiltrating CD45 cells versus proteinuria levels in 6- to 7-month-old Fc $\gamma$ RIIb-KO control mice $(n=56)$. Spearman's $r=0.624$; ${ }^{* * * *} P<0.0001$.

cytometric measurements confirmed the histological results and showed a marked reduction in the number of infiltrating leukocytes, mainly CD11 $\mathrm{b}^{+}$cells and $\mathrm{T}$ cells, in infected FcyRIIb-KO compared with uninfected control kidneys (Figure 4B and Supplemental Figure 4A). We found a correlation between the number of infiltrating leukocytes and proteinuria scores (Spearman's $r=$ $0.624, P<0.0001$, Figure $4 \mathrm{C}$ ), suggesting a contribution of infiltrating leukocytes to the kidney pathology.

Although malaria infection protected the kidney, it did not reduce infiltration into the liver: $\mathrm{H} \& \mathrm{E}$ staining showed similar levels of leukocyte accumulation in Fc $\gamma$ RIIb-KO liver, regardless of infection status (Figure 5A). Flow cytometric analysis confirmed that every immune population tested was equally present in malaria-infected and uninfected Fc $\gamma$ RIIb-KO livers. The same analysis in kidney showed a reduction in the absolute numbers of every $\mathrm{CD} 45^{+}$cell population in infected animals compared with controls (Figure 5, B and C). Population frequency calculations showed that CD11b ${ }^{+}$was a primary cell type reduced in kidney parenchyma of infected mice, however malaria did not alter this population in kidney-associated vasculature (Figure 5D and Supplemental Figure 4A). We noticed that the types of infiltrating leukocytes were different in kidney compared with liver. Kidney-infiltrating leukocytes were predominantly $\mathrm{T}$ cells $\left(\mathrm{CD}^{+}\right.$and $\left.\mathrm{CD} 8^{+}\right)$and macrophages $\left(\mathrm{F} 4 / 80^{+}\right)$, whereas the liver included large numbers of $\mathrm{B}$ cells in addition to $\mathrm{T}$ cells, but no macrophages (Figure 5, C and D, and Supplemental Figure 4A).

Spleens and axillary and inguinal lymph nodes (LNs) showed no differences in cell numbers 4 months after being infected or not with $P$ y. In contrast, renal LN counts were significantly reduced in infected mice compared with counts in control mice, primarily due to a reduction in $B$ cell numbers (Figure $5, E-G$, and Supplemental Figure 4B). Overall, these results demonstrate that Plasmodium infection prevented kidney-specific immune cell infiltration and expansion of 
A
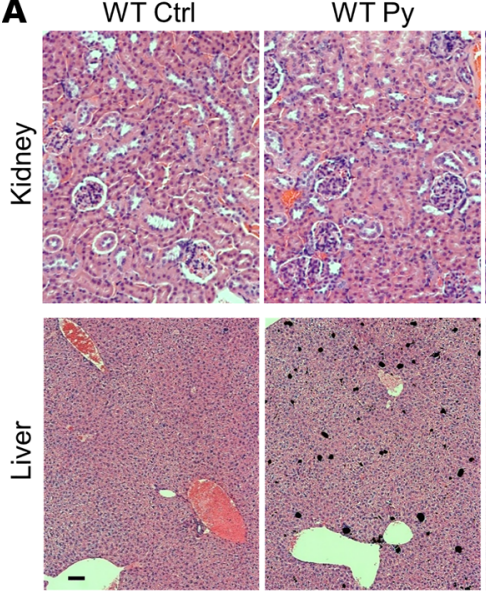

FcyRllb[KO] Ctrl FcyRllb[KO] Py
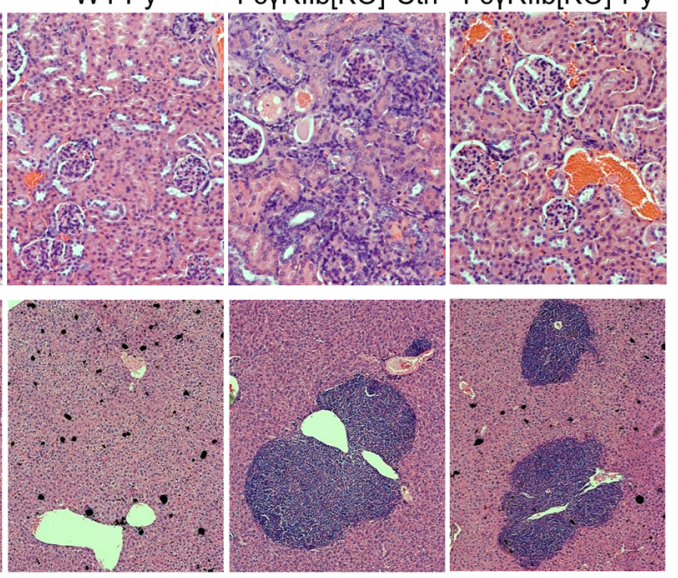

B
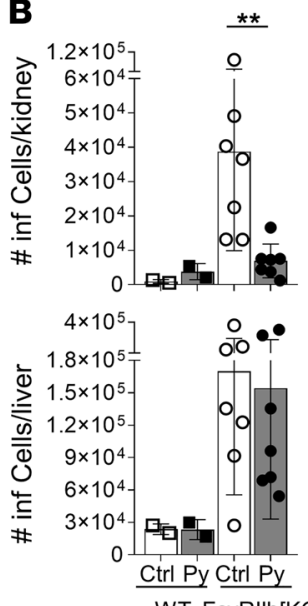

WT FcyRIIb[KO]

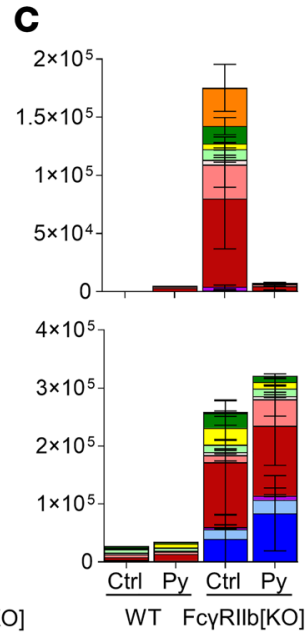

D

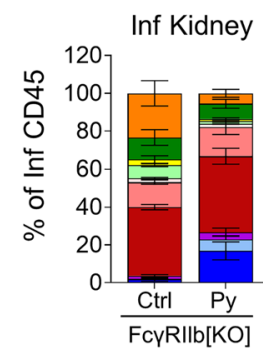

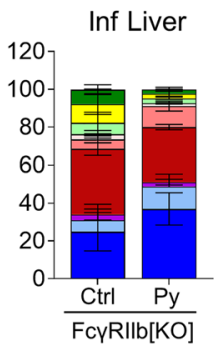
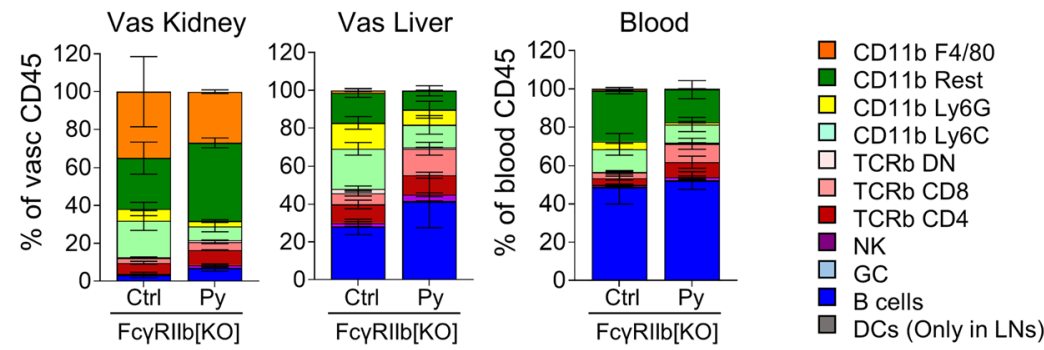

E

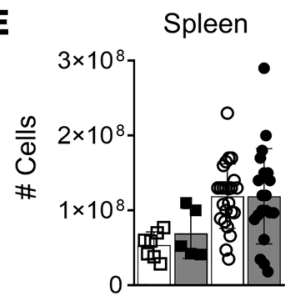

$\mathbf{F}$

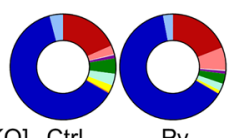

Axillary LN
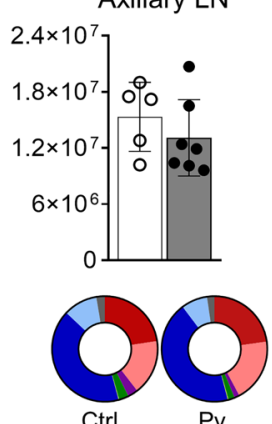

Inguinal LN
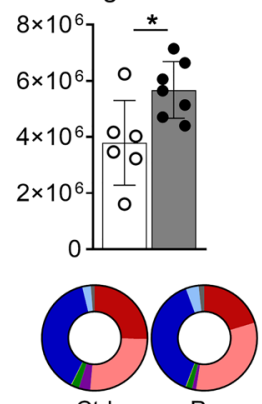

Ctrl
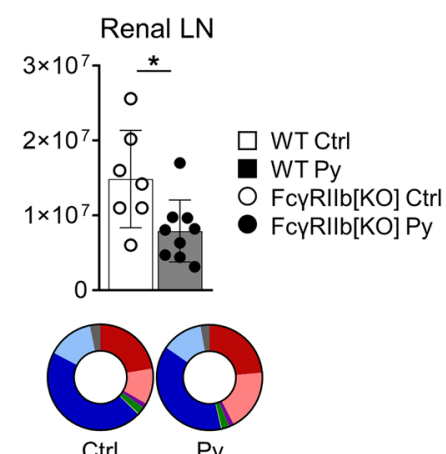

G

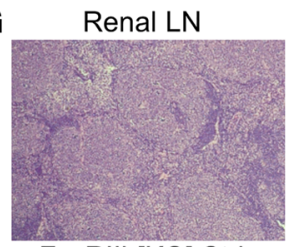

FcyRIIb[KO] Ctrl

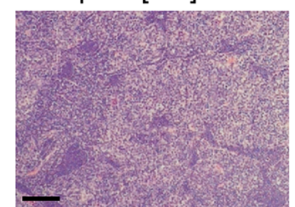

FcyRIIb[KO]Py

Figure 5. The protective effect of parasite infection is kidney specific. (A-D) Kidney and liver analysis in WT and Fc $\gamma$ RIllb-KO mice 5 months after infection with Py. (A) Representative H\&E-stained images of kidney and liver infiltration in mice of the indicated genotype and infection status (scale bar: 100 $\mu \mathrm{m})$. (B) Flow cytometric quantification of infiltrating leukocytes per kidney or liver sample. $n=2$ and $n=7$ for WT and Fc $\gamma$ RIlb-KO groups. (C) Quantification of absolute number of leukocytes belonging to various immune cell subpopulations infiltrating kidney and liver. $n=1$ and $n=3$ for WT and Fc $\gamma R I I b-K O$ groups. (D) Frequencies of leukocyte subpopulations among total infiltrated or vasculature CD45+cells in kidney or liver, as well as in peripheral blood $(n=$ 3). GC, germinal center B cells. (E) Absolute number of cells in spleen and LNs from Fc $\gamma R$ Illb-KO mice. Spleen: $n=7,5,24$, and 20 for WT control, WT Py-infected, FcyRIIb-KO control, and FcyRIIb-KO Py-infected mice, respectively. Axillary LNs: $n=5$ and $n=7$; inguinal LNs: $n=6$ and $n=7$; renal LNs: $n=7$ and $n$ = 9, for control and $P y$-infected mice, respectively. Cell subpopulations were detected by flow cytometry (as shown in Supplemental Figure 4) and are col-

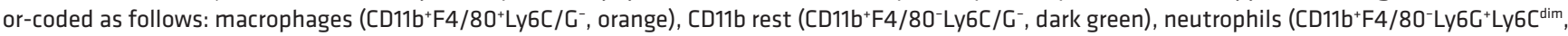

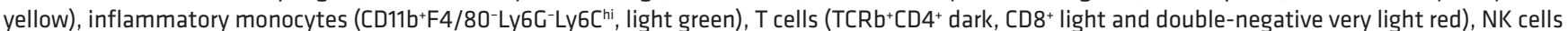
(TCRb-NK1.1+CD49b', purple), B cells (B220+, blue), germinal center B cells (B220+CD95 ${ }^{+} C L-7^{+}$, light blue), and DCs (CD11 $\mathrm{C}^{+} \mathrm{MHC}-\mathrm{II} I \mathrm{~A} / \mathrm{IE} \mathrm{E}^{\mathrm{hi}}$, gray). (F) Frequencies of leukocyte subpopulations in the specified organ and genotype $(n=3)$. (G) H\&E staining of renal LNs from infected or control Fc $\gamma \mathrm{RIIb}-\mathrm{KO}$ mice $(s c a l e$ bar: $100 \mu \mathrm{m})$. Group comparisons between Fc $\gamma$ RIIb-KO were made using the Mann-Whitney $U$ test. ${ }^{*} P<0.05$ and ${ }^{* *} P<0.01$.

renal LNs, but it did not affect leukocyte infiltration of liver or immune cell populations in the spleen or in LNs not associated with the kidney.

CCL17 expression is uniquely reduced in kidney following Plasmodium infection. We tested the effect of infection with $P y$ on the expression of several genes associated with kidney pathology in SLE, separating $\mathrm{CD}^{-} 5^{-}$resident nonimmune cells from $\mathrm{CD} 45^{+}$immune cells in the kidney. Quantitative PCR (qPCR) performed on purified kidney cells showed that the infection did not alter mechanisms that have been previously linked to lupus nephritis, such as ferroptosis (regulated by Aco1, a.k.a. IRP1) $(27,28)$ or hypoxia (regulated 

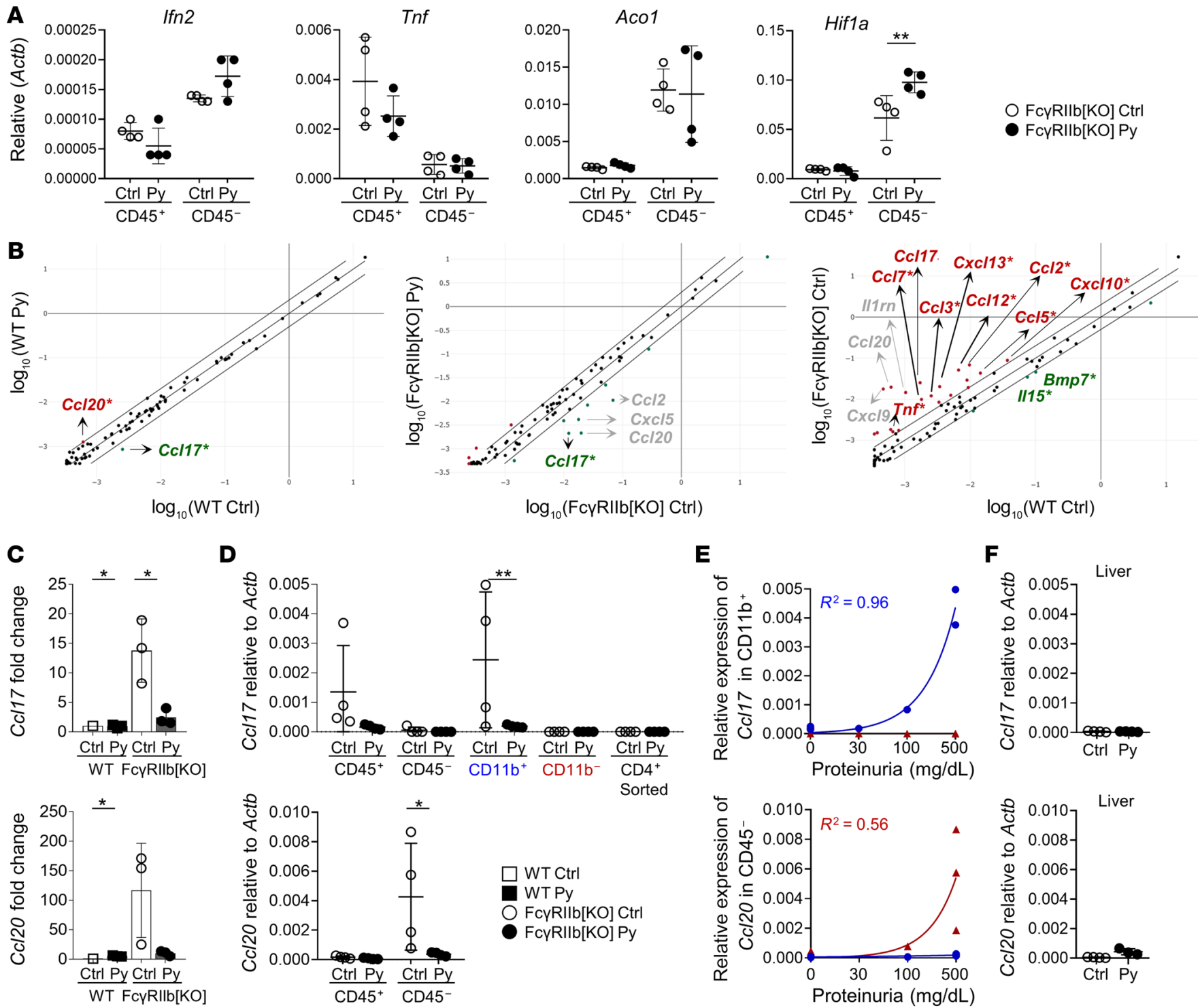

Figure 6. Parasite infection reduces the leukocyte-attracting chemokines CCL20 and CCL17 in the kidney without affecting innate cytokines and chemokines. (A-E) Gene expression studies in kidneys of FcyRllb-KO mice 5 months after infection with Py. (A) mRNA expression of the indicated genes in bead-purified kidney leukocytes $\left(C D 45^{+}\right)$and nonimmune cells $\left(C D 45^{-}\right)$. Results are relative to Actb expression $(n=4)$. (B) Gene expression analysis of total kidney RNA using the Cytokines \& Chemokines RT ${ }^{2}$ Profiler PCR Array kit. Scatter plots show the fold change between WT infected versus control, Fc $\gamma$ RIIb-KO infected versus control, and WT versus Fc $\gamma$ RIIb-KO mice. Red and green colors represent higher or lower expression compared with the control $(n=3)$. (C) Resulting fold change in expression of $C c / 17$ and $C c / 20$ genes compared with expression in WT control mice. (D) Expression of the indicated chemokines in leukocytes (CD45+), myeloid (CD11b+) cells, sorted infiltrating CD4+ T cells, and kidney nonimmune cells (CD45) $(n=4)$. (E) Graphs showing the correlation of Cc/17 expression in CD11b+ cells and Cc/20 expression in $\mathrm{CD}_{4} 5^{-}$cells to proteinuria levels in Fc $\gamma \mathrm{RIIb}-\mathrm{KO}$ control mice $\left(R^{2}=0.96\right.$ and 0.56 , respectively). (F) Liver expression of the indicated chemokine genes $(n=4)$. Data are presented as the mean \pm SD. Group comparisons were made using $1-$ and 2 -way ANOVA. ${ }^{*} P<0.05$ and ${ }^{* *} P<0.01$.

by Hifla) (9-11), or the levels of type I IFN or TNF (29) (Figure 6A). We next used the Cytokines \& Chemokines $\mathrm{RT}^{2}$ Profiler PCR Array kit to compare kidneys of previously infected Fc $\gamma$ RIIb-KO mice with control kidneys as a measure of the ongoing pathology (Figure 6B). Ccl17 was the only transcript that was significantly reduced in the $P y$-infected samples compared with transcript levels in controls in both WT and Fc $\gamma$ RIIb-KO kidneys. Other transcripts were found to be significantly elevated in Fc $\gamma$ RIIb-KO compared with WT kidneys (Ccl2, Ccl3, Ccl5, Ccl7, Ccl12, Ccl13, Cxcl10, Cxcl13, Tnf). However, they were not significantly altered by malaria infection. Two of them
(Ccl2O and Ccl2) had a nonsignificant trend of lower expression in malaria-infected Fc $\gamma \mathrm{RIIb}-\mathrm{KO}$ kidneys compared with expression in the uninfected controls (Figure 6B). We verified and expanded these results by expression analysis of individual chemokines, focusing on $\mathrm{Ccl} 17$ and $\mathrm{Ccl} 2 \mathrm{O}$ as the 2 prominent transcripts that showed a reduction in infected conditions (significantly in the case of Ccl17 and trending, but not at a highly significant level, in the case of Ccl20).

Individual assessment by qPCR confirmed increased expression of Ccl17 and Ccl2O in FcrRIIb-KO compared with WT kidney, and both chemokines were reduced in $P y$-infected samples 
A

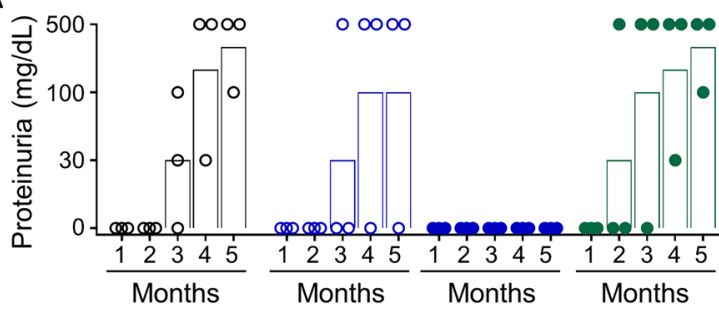

B

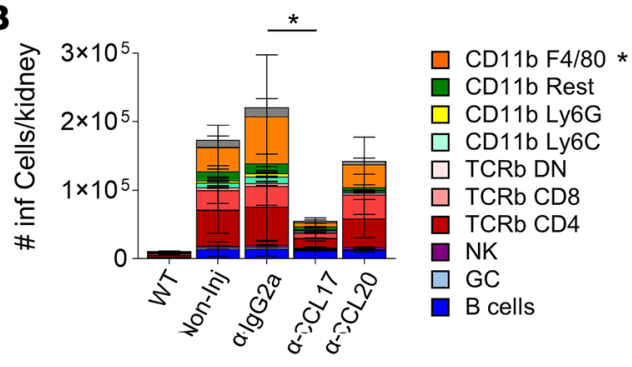

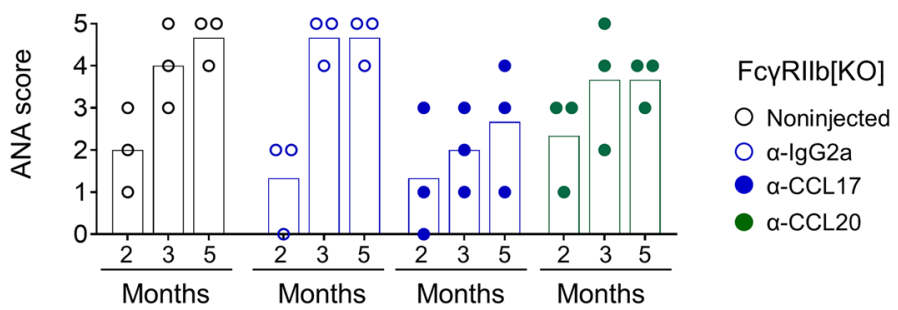
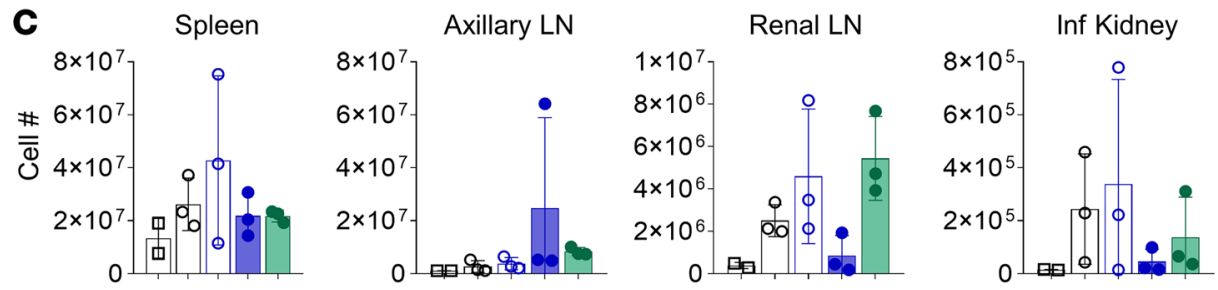

WT noninjected

O FcyRllb[KO] noninjected

O FcyRIIb[KO] $\alpha-\operatorname{lgG} 2 a$

- FcyRIIb[KO] $\alpha-C C L 17$

- FcyRIIb[KO] $\alpha-C C L 20$
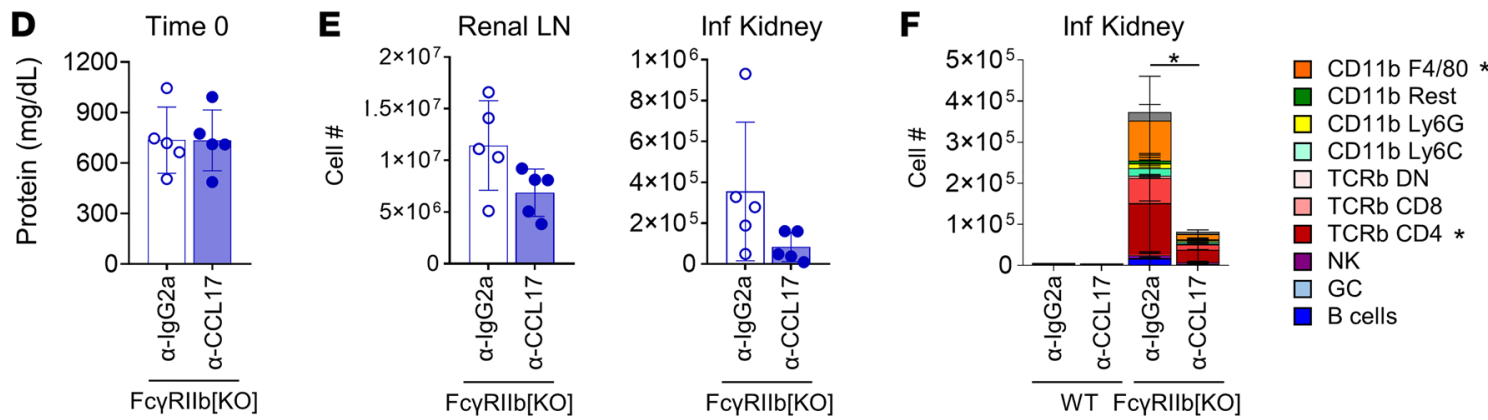

Figure 7. Depletion of the chemokine CCL17 reduces severe glomerulonephritis. (A-C) Long-term depletion of CCL17 and CCL20 chemokines in Fc $\gamma \mathrm{RIIb}-\mathrm{KO}$. Two-month-old mice were injected i.v. with $20 \mu \mathrm{g}$ IgG2a (isotype control) or CCL17 or CCL20 antibodies, 3 times per month for the duration of the experiment. (A) Proteinuria levels and ANA scores monthly after the first injection $(n=3)$. ANA scores were determined by positive lgG staining in different serum dilutions: ANA score of 1 (1:100), ANA 2 (1:300), ANA 3 (1:900), ANA 4 (1:2700), and ANA 5 (1:8100). (B) Number of infiltrating cells in the kidneys 5 months after the first injection. (C) Number of cells in the spleen, axillary LNs, renal LNs and infiltrated into the kidneys for all groups. $n=2$ for WT mice and $n=3$ in the indicated FcyRIIb-KO injected mice. (D-F) Short-term depletion of CCL17 in Fc $\gamma$ RIlb-KO mice with ongoing glomerulonephritis. Mice at 7.5 months of age were injected i.v. with $25 \mu \mathrm{g}$ IgG2a or CCL17 on days $0,4,8$, and 12. (D) Mice were selected on the basis of similar proteinuria levels, as measured by QuantiChrom colorimetric assay. (E) Number of cells in renal LNs and infiltrated into the kidney. (F) Subpopulations of cells detected by flow cytometry on day 18 . $n=1$ for WT and $n=5$ for Fc $\gamma R$ IIlb-KO groups. Data are presented as the mean \pm SD. Group comparisons were made using 1 - and 2 -way ANOVA. ${ }^{*} P<0.05 . \alpha$, anti.

compared with uninfected ones (Figure 6C). We found that Ccl17 was primarily expressed by infiltrating immune cells $\left(\mathrm{CD} 45^{+}\right)$and that $\mathrm{Ccl} 20$ was expressed mostly in $\mathrm{CD}^{-} 5^{-}$kidney cells (Figure 6D). Ccl17 expression was limited to CD11 $\mathrm{b}^{+}$cells and absent in the $\mathrm{CD}^{+} \mathrm{T}$ cell population in the kidneys. Expression of either of these 2 chemokines in kidney was correlated with proteinuria levels, exceptionally well in the case of Ccl17 (Figure 6E). Neither Ccl17 nor Ccl2O was expressed in liver (Figure 6F).

Depletion of CCL17, but not CCL2O, protects against glomerulonephritis. To determine whether these chemokines have a role in the development of severe lupus glomerulonephritis, we injected specific antibodies against CCL17, CCL2O, or an IgG2a isotype control and tested disease progression. Long-term depletion of CCL17 starting with young Fc $\gamma$ RIIb-KO mice resulted in the elimination of proteinuria and leukocyte infiltration into the kidney, whereas CCL2O depletion or the isotype antibody injection had no effect (Figure 7, A and B). As was the case in Plasmodium-infected mice, the protection conferred by injection of anti-CCL17 antibodies was specific to the kidneys and renal LNs, with no effects on the spleen axillary LN inflammation, or circulating autoantibody levels (Figure 7, A and C). We then performed a short-term CCL17 depletion experiment in older animals that had ongoing nephritis symptoms. Experimental and control FcyRIIb-KO mice were matched to start the experiment with similar levels of proteinuria (Figure 7D) and were then injected with anti-CCL17 
A

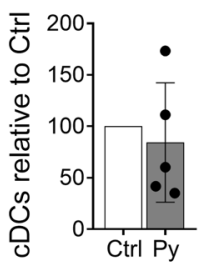

C

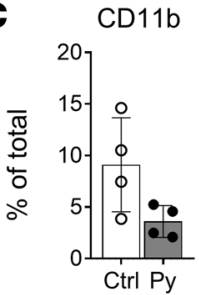

$\mathbf{F}$
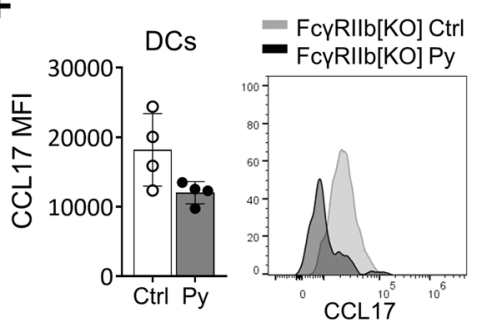

G

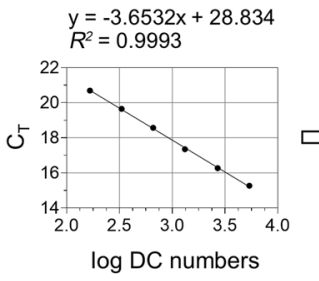

B
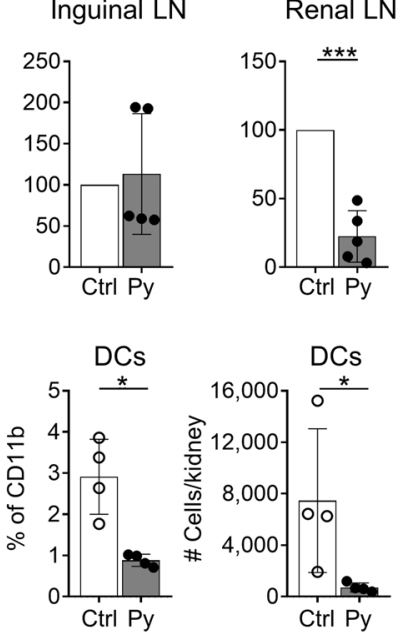
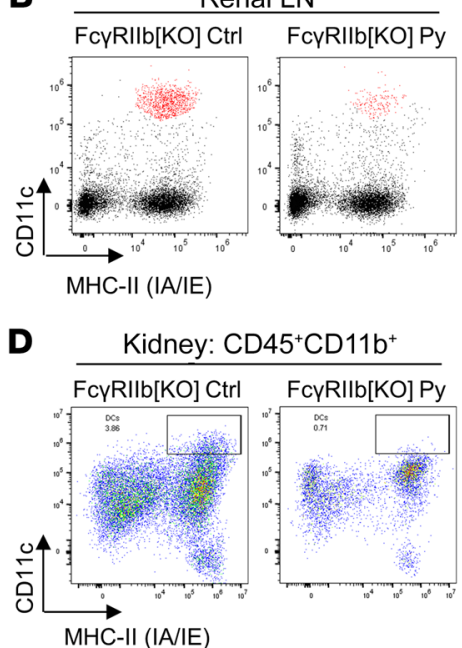

E
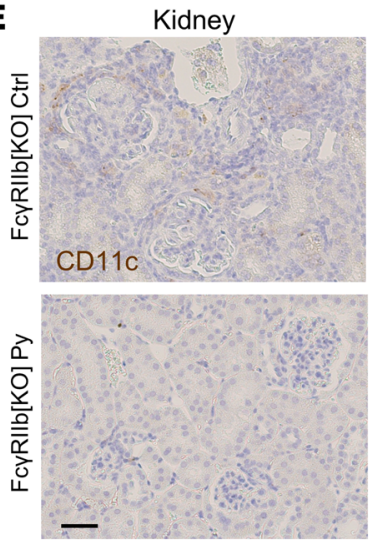

O FcyRIIlb[KO] Ctrl

- FcyRllb[KO] Py

I
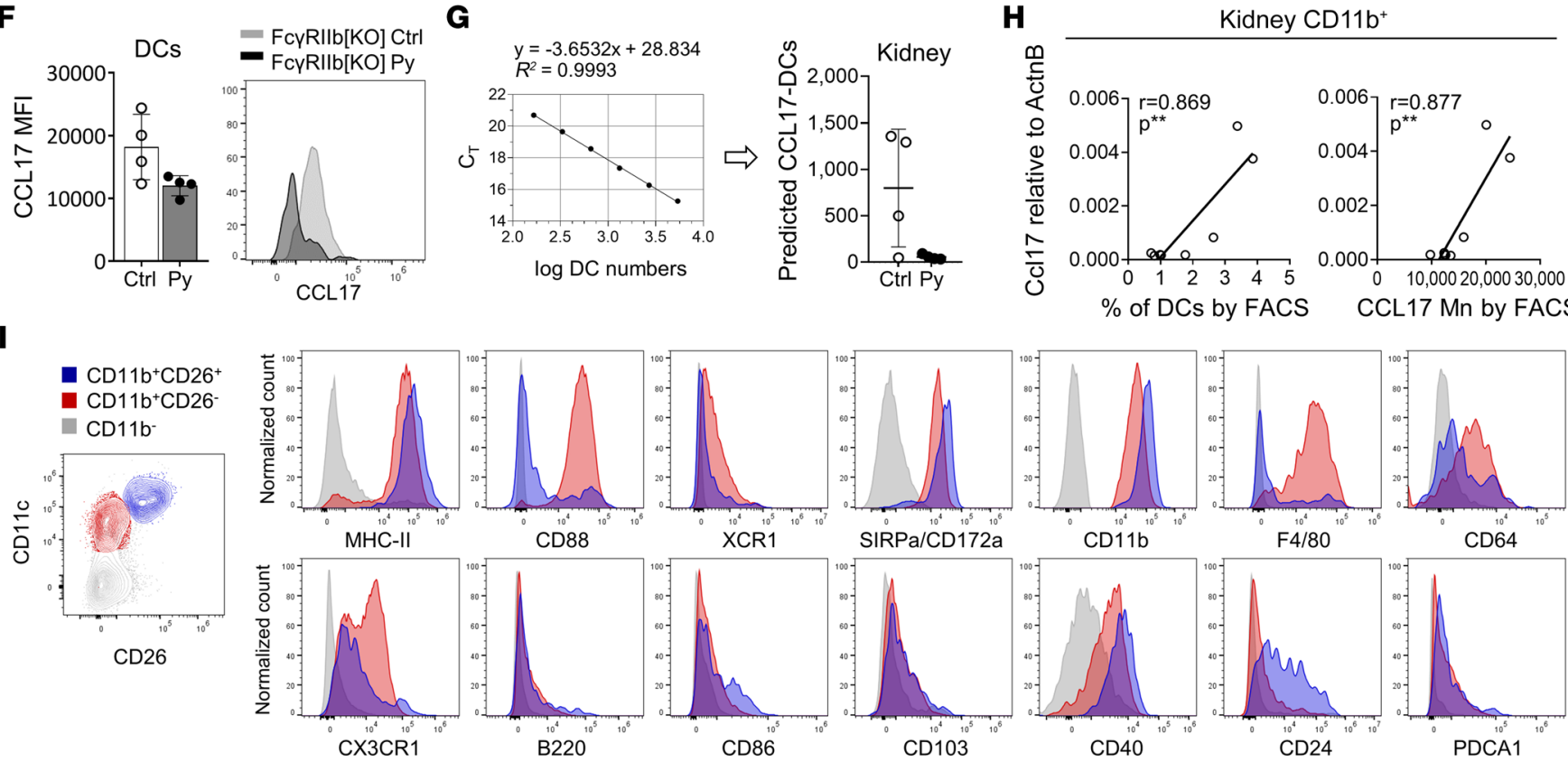

Figure 8. Parasite infection inhibits kidney disease by reducing CCL17-expressing DCs. Analysis of DCs from Fc $\gamma \mathrm{RIIb-KO}$ mice 5 months after infection with Py. (A) Number of conventional DCs (CDCs) (CD11b+CD11 chi MHC- $\left.\mathrm{II}^{+}\right)$in the spleen and axillary, inguinal, and renal LNs relative to numbers of $\mathrm{DCs}$ from Fc $\gamma \mathrm{R}$ IIb-KO controls $(n=5)$. (B) Dot plots showing the gating of DCs (in red) in renal LNs. (C) Flow cytometric measurements of DCs in the

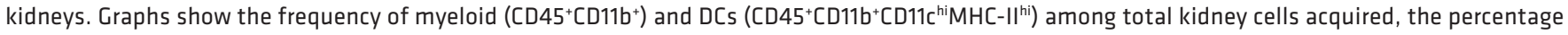
of DCs among CD11 b+ cells, and the number of DCs in the kidneys $(n=4)$. (D) Representative dot plot showing the gating of DCs within the kidney-infiltrating CD11 b+ cell population. (E) CD11c staining in kidney sections from the indicated mice (scale bar: $200 \mu \mathrm{m}$ ). (F) Intracellular expression of CCL17 measured in CD11 ${ }^{\text {hi }}$ kidney-infiltrating DCs $(n=4)$. Fc $\gamma R$ IIlb-KO controls (light gray); expression in samples after Py infection (dark gray). (C) Correlation of Cc/17 expression measured by qPCR with the number of DCs. Left: The regression curve was obtained with serial dilutions of BM-derived DCs grown in culture. Right: Estimate of the number of DCs that theoretically corresponded to the CCL17 levels detected in the kidney of mice with the indicated genotype and infection status $(n=4) . C_{T}$, cycle threshold in CCL17 mRNA qPCR testing. (H) Correlation graphs showing Ccl7 gene expression levels in CD11 b+ cells versus the percentage of DCs or expression of CCL17 measured by flow cytometry in the same Fc $\gamma$ RIIb-KO control mice (Pearson's $r=0.869$ and 0.877 , 2-tailed approximate $P$ value $\left.{ }^{* *} P<0.01, n=8\right)$. (I) Surface marker characterization of kidney DCS (CD11b $\left.{ }^{+} C D 11 c^{\text {hic }} C D 26^{+}\right)($blue), macrophages (CD11 $\left.\mathrm{b}^{+} \mathrm{CD} 11 \mathrm{c}^{+} \mathrm{CD} 26^{-}\right)$(red), and CD11b- cells (gray). Data are presented as the mean \pm SD. Group comparisons were made using a 1-sample $t$ test and the Mann-Whitney $U$ test. ${ }^{*} P<0.05$ and ${ }^{* * *} P<0.001$.

antibodies for 2 weeks. By day 18, we observed that mice that had received injections of CCL17-blocking antibodies had decreased renal LN inflammation as well as a reduced number of infiltrated cells in the kidneys (Figure 7, E and F). These results confirm the important role of this chemokine in severe glomerulonephritis.
CCL17-expressing inflammatory DCs are correlated with end-stage kidney nephritis. CCL17, also known as TARC, is highly expressed in thymus and DCs and has been associated with Th2 responses and chemotactic activity for $\mathrm{T}$ cells but not monocytes or granulocytes. CCL17-expressing DCs have been found in external barriers of the 
A

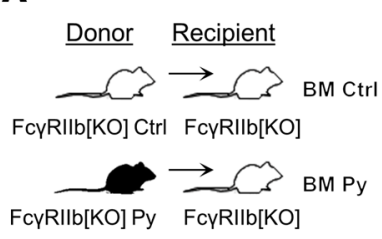

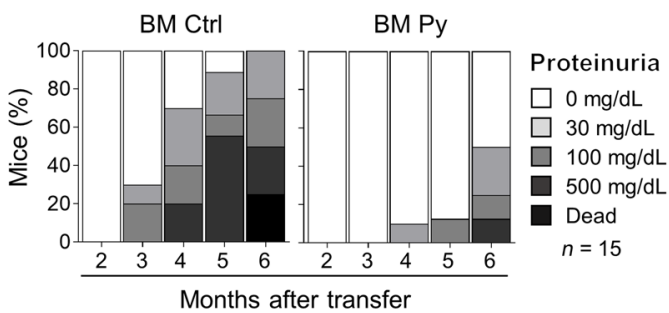

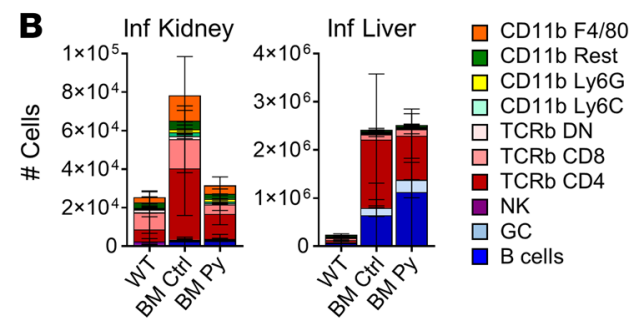

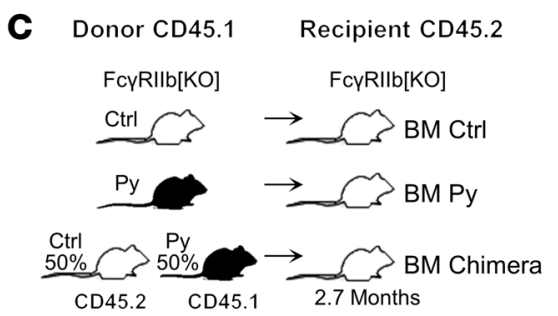
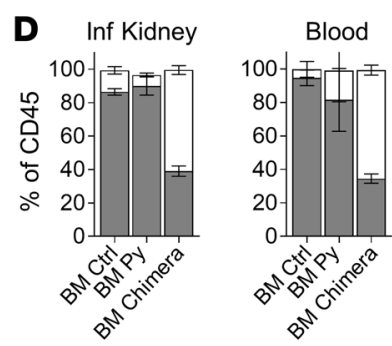

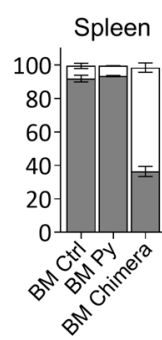

$\square \mathrm{CD} 45.1$

$\square \mathrm{CD} 45.2$
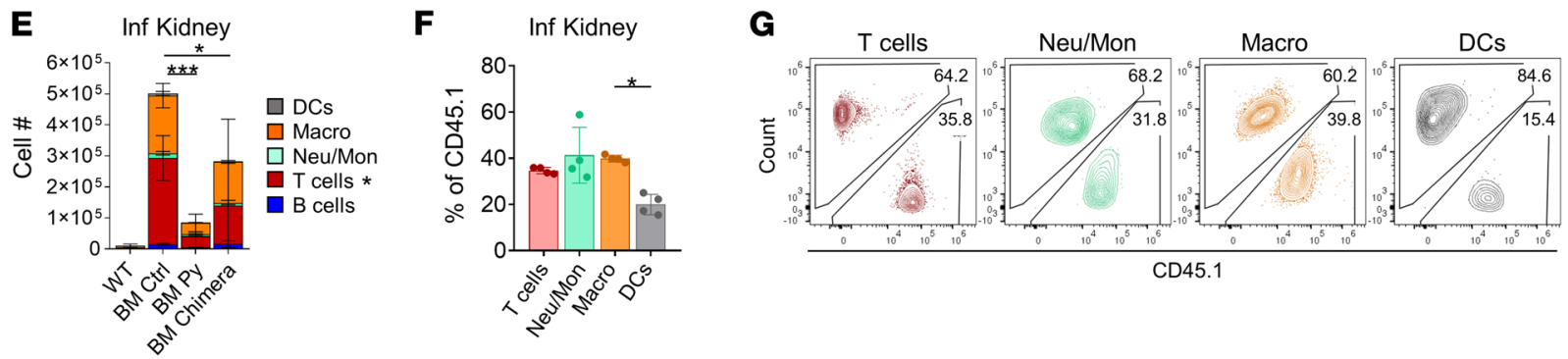

Figure 9. BM alterations underlie the protective effect of parasite infection. (A and B) Data obtained following BM adoptive transfer experiments. Mice received BM cells from Fc $\gamma$ RIIlb-KO uninfected (BM Ctrl) or infected (BM Py) donors. (A) Percentage of mice with various levels of proteinuria as indicated in grayscale. Dead mice are shown in black. $n=14$ (months 2-4) and $n=12$ (months 5-6). (B) Absolute number of infiltrating leukocytes in the kidney or liver, with each immune cell population indicated by the color scheme, 5 months after BM transfer. $n=2$ and $n=5$ for the WT and Fc $\gamma R$ RIlb-KO groups. (C-C) Chimera assay. Data were obtained following BM adoptive transfer experiments indicated in the scheme in C. Mice received BM cells from Fc $\gamma$ RIlb-KO CD45.1 uninfected or infected donors or Py/control mixed chimeric mice (BM chimera). (D) Graphs show the reconstitution of donor cells (CD45.1) in the indicated organs. (E) Number of infiltrating leukocyte subpopulations in the kidneys 6 months after BM transfer. $n=2,3,2$, and 4 for WT, BM control, Py-infected,

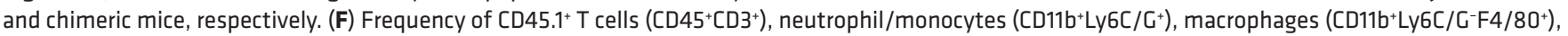
and DCs (CD11 $\left.\mathrm{b}^{+} \mathrm{Ly} 6 \mathrm{C} / \mathrm{G}^{-} \mathrm{F} 4 / 80^{-} \mathrm{SSC}^{\circ}\right) . n=4$. (C) Flow cytometry gating strategy. Data are presented as the mean \pm SD. Group comparisons were made using 1- and 2-way ANOVA. ${ }^{*} P<0.05$ and ${ }^{* *} P<0.001$. Macro, macrophages; Neu/Mon, neutrophils/monocytes.

skin and mucosa but not in the spleen (30). We observed a reduction in the number of DCs (measured as CD11 $\mathrm{c}^{+} \mathrm{MHC}-\mathrm{II}^{\text {hi) }}$ after Py infection in renal LNs, but not in the spleen or axillary or inguinal LNs, 5 months after infection (Figure 8, A and B). Detailed flow cytometric analysis of kidney $\mathrm{CD} 45^{+}$cells revealed the absence of a DC population (CD11 $\left.\mathrm{c}^{\text {hi }} \mathrm{MHC}-\mathrm{II}^{\text {hi) }}\right) 5$ months after $P y$ infection that was apparent in FcyRIIb-KO controls (Figure 8B). This reduction was proportionally more pronounced in DCs than in the broader CD11 $\mathrm{b}^{+}$ cell population (Figure 8, C and D). Histological analysis of CD11c antibody staining of kidney sections revealed that DCs were present in periglomerular and interstitial areas of Fc $\gamma$ RIIb-KO kidneys but absent in $P y$-infected kidneys (Figure 8E).

We measured the intracellular expression of CCL17 in CD11 $\mathrm{c}^{\text {hi }}$ kidney cells and found a reduction of CCL17 in FcyRIIb-KO infected mice (Figure 8F). In addition, Ccl17 transcript expression levels measured in kidneys of Fc $\gamma$ RIIb-KO mice were comparable to the estimated Ccl17 expression levels from the same number of cells $\left(1 \times 10^{3}\right.$ to $\left.2 \times 10^{3}\right)$ raised as BM-derived DCs in vitro (Figure $8 G$ ). Measures of the proportion of DCs within the myeloid population in the kidney, as well as the inten- sity of CCL17 staining in DCs by flow cytometry, were highly correlated with the levels of $\mathrm{C} c l 17$ measured by PCR in the kidney (Figure $8 \mathrm{H}$ ). These results strongly suggest that infiltrating DCs are the sole source of CCL17 chemokine present in kidneys of Fc $\gamma$ RIIb-KO mice. To ensure that the CD11 $\mathrm{c}^{+}$cells we detected were truly DCs and to distinguish them from inflammatory macrophages, we fully characterized them by surface marker expression: these cells were $\mathrm{CD} 26^{+} \mathrm{CD} 88^{-\mathrm{XCR}} 1^{-\mathrm{CD}} 172 \mathrm{a}^{+} \mathrm{CD} 64^{+}$, thus resembling the inflammatory type 2 DCs originally described in lung inflammatory conditions by the Lambrecht group (18) (Figure 8I). These DCs also expressed $\mathrm{CD}_{24}{ }^{+}$and were negative for F4/80, showing clear, distinct expression markers compared with kidney-infiltrating macrophages $\left(\mathrm{CD} 26^{-} \mathrm{CD} 88^{+} \mathrm{F} 4\right.$ $80^{\mathrm{hi}} \mathrm{CD} 24^{-}$), which were still detected in Py-infected kidneys, albeit in smaller numbers (MHC-II+ cells in Figure 8D).

$B M$ alterations with a specific impact on DCs underlie the kidney-protective effect of a parasitic infection. To assess whether Py-induced alterations in BM cells were the cause of the reduced kidney infiltrates, we reconstituted irradiated Fc $\gamma$ RIIb-KO mice with BM cells from FcyRIIb-KO mice previously infected with $P y$ or with 
BM from uninfected Fc $\gamma$ RIIb-KO mice (controls). We verified that the infection did not emerge in the recipient mice (Supplemental Figure 5A), and the reconstitution was done successfully (Supplemental Figure 5B). We then followed systemic and tissue-specific autoimmune symptoms for several months. Mice reconstituted with control BM developed full autoimmune disease, including glomerulonephritis and proteinuria. In contrast, mice reconstituted with $P y$-infected $\mathrm{BM}$ had significantly lower levels of proteinuria and $\mathrm{T}$ cell and monocyte infiltration in the kidneys (but not in the liver) than did control mice (Figure 9, A and B, and Supplemental Figure 5, C-E). Other measures of systemic autoimmunity, including splenomegaly, serum ANAs, and inflammatory cytokine levels were all comparable among recipients of $P y$-infected $\mathrm{BM}$ or control BM (Supplemental Figure 5, F-H). Thus, the protective effect of malaria infection on lupus nephritis could be traced to alterations of BM cells. This indicated that a BM-derived, CCL17-expressing cell population might be absent in $P$ y-infected kidneys and present in kidneys from $\mathrm{Fc} \gamma \mathrm{RIIb}-\mathrm{KO}$ controls.

To determine whether the BM alterations induced by $P y$ infection were targeting kidney-infiltrated DCs specifically, we generated chimeras with a 1:1 mix of BM from $P y$-infected and uninfected donors (Figure 9C). BM reconstitution in this condition resulted in approximately $90 \%$ leukocytes derived from donor cells in every tissue tested, as was evident in recipient mice reconstituted with unmixed control BM (Figure 9D). Recipients of a 1:1 mix of Py/ control BM had approximately $40 \%$ of reconstituted leukocytes derived from $P y$-infected BM (CD45.1) and 60\% derived from control BM (CD45.2) systemically (Figure 9D). Analysis of local kidney-infiltrating cell populations confirmed that mice reconstituted with $P$-infected BM averted immune cell infiltrates in the kidneys, while those reconstituted with control BM developed full pathology (Figure 9E). Out of all the CD $45^{+}$cell populations present in the kidneys of reconstituted 1:1 chimeric mice, DCs were the ones primarily biased against those of the CD $45.1^{+}$allotype, which marked cells derived from $P$-infected BM. In contrast, macrophages, neutrophils, and T cells derived from infected BM infiltrated the kidneys comparably to those derived from uninfected BM. It is worth noting that $\mathrm{T}$ cells, neutrophils/monocytes, and macrophages had the same proportional representation ( 60\% CD $45.2^{+}$ vs. $40 \% \mathrm{CD} 45.1^{+}$) as in other organs (Figure 9D), whereas DCs in kidneys had proportionally and substantially fewer CD $45.1^{+}$cells (15\%) (Figure 9, F and G). These results suggest that Plasmodium infection induced long-term alterations in BM cells that specifically hindered the ability of DCs to infiltrate the kidney.

\section{Discussion}

Here, we report the finding that kidney-infiltrating type 2 DCs were highly correlated with end-stage kidney nephritis in lupusprone Fc $\gamma$ RIIb-KO mice. We have defined the role of CCL17-producing inflammatory DCs in this model of lupus nephritis by taking advantage of the protective effect of infection with $P y$, which we found specifically targeted kidney immune cell infiltrates. The observed differential effects between previously $P y$-infected and uninfected FcyRIIb-KO mice point to DCs as critical elements in the transition from glomerular inflammation to a more destructive intratubular infiltration. Infection with Plasmodium was long ago reported to increase survival in the NZB/W lupus model (20), and autoreactive antibodies were detected in populations infected with malaria parasites in multiple studies $(21,31,32)$. It was speculated that the parasite confers a certain level of protection against destructive inflammation, albeit with an unknown mechanism (33). Our experiments with Fc $\gamma$ RIIb-KO confirm the finding that infection with Plasmodium induces systemic autoreactivity while protecting from lethal nephritis. We now show that the malaria parasite specifically prevented end-stage kidney disease without altering systemic autoimmune inflammation or local innate pathways (IFN-I, TNF) in kidneys. Lupus-prone mice infected with Plasmodium developed the same spontaneous immune system hyperactivation, with equivalent expansion of activated lymphocytes, splenomegaly, lymphadenopathy, and high serum IgG titers as the uninfected controls. This is in contrast to a previous report that had suggested a reduction of B cell activity following malaria infection (34). Moreover, Plasmodium infection in our studies did not fundamentally alter the autoantibody specificity present in Fc $\gamma$ RIIb-KO mice (IgG or IgM). A handful of autoantibodies increased in infected mice compared with uninfected Fc $\gamma$ RIIb-KO mice. These enhanced specificities did not confer protection in serum passive transfer experiments, arguing against the possibility that infection with Plasmodium might induce the expression of some type of tolerogenic factor measurable in the circulation, and in contrast with an earlier report that described the beneficial effect on NZB/W mice that received polyclonal IgGs from malaria-infected BALB/c mice (25).

IC deposition in kidney is considered the triggering factor leading to lupus nephritis, causing a cascade of complement, Fc receptor, and myeloid cell activation that leads to chemokine production and recruitment of pathogenic leukocytes $(1,2)$. Our results of differential effects after infection argue for a regulatory step separating glomerular inflammation triggered by IC deposition and the more destructive step of cellular infiltration. Infection with Plasmodium precluded leukocyte infiltration in kidney parenchyma but did not prevent proliferative and mesangial glomerulonephritis induced by IC deposition and C3 fixation. In contrast, liver-infiltrating leukocytes were equivalent in numbers, regardless of infection status. Evaluation of the infiltrating cell populations in kidney and liver suggested a different mechanism driving the infiltration: $\mathrm{B}$ and $\mathrm{T}$ lymphocytes predominated in liver-infiltrating cell populations, while T cells, monocytes, and DCs predominated in kidney infiltrates. Even more evident is the distinction we observed between kidney parenchyma, which included more $\mathrm{T}$ cells and macrophages, versus the kidney-associated vasculature, which harbored more varied monocyte populations, suggesting that kidney pathology is accompanied by active recruitment of selected cell populations as opposed to a mere vessel wall disruption, which might better describe the process in liver. Along with this, infection with $P y$ only reduced the inflammatory state of the renal LNs, without an alteration in the spleen or axillary and inguinal LNs. All these data pointed to a specific prevention of kidney destruction by limiting the active recruitment of inflammatory cells that, as our experiments have established here, were of BM origin.

Prevention of pathogenic infiltrates would alleviate the most damaging effects, as renal infiltration of mononuclear cells has been reported to be associated with a poor prognosis in patients 
with SLE (1). In mice, a detailed analysis of infiltrates in the NZB/W lupus model showed that a mix of macrophage/DC infiltrates correlated with severe kidney disease, and, more specifically, infiltrating CD11chi DCs were detected after proteinuria onset (14). Our results point to a malaria-dependent prevention of DC infiltration that might be key in turning an initial innate response triggered by IC deposition into an adaptive response that requires DCs to perhaps prime and recruit $\mathrm{T}$ cells to local sites. Our detailed flow cytometric analysis characterized these cells as inflammatory type 2 DCs, as defined by the Lambrecht group (18). These cells would be capable of presenting local antigens derived from the injured kidney, suggestive of a damaging adaptive response targeting kidney-specific structures, also consistent with the specific effect observed in kidney-draining LNs compared with other LNs. Because this type of inflammatory DCs have a hybrid expression profile but with a marked type 2 bias (18), it is conceivable that they would be less likely to arise after the very strong type 1 inflammatory response elicited by the malaria parasite $(23,35)$. The long-term and BM-mediated protective effects of the parasite argue for a possible epigenetic change or modification of BM precursors that would impede the development of these cells for several months.

One indication that DCs are a target of a malaria protective effect was the unique effect on expression of the DC-specific chemokine CCL17, which was the only gene in our cytokine array that was significantly lower in the kidney-infiltrating immune cells of infected versus control mice. In addition, expression of CCL2O was reduced in nonimmune kidney cells of malaria-infected mice compared with those of controls, and thus was a good indicator of ongoing tissue damage. CCL2O has been extensively studied in the context of SLE and found to correlate with prognosis (15). Meanwhile, CCL17 has not been directly studied in the mediation of lupus glomerulonephritis, although it is included in the overall data from recent single-cell expression studies of kidney glomerulonephritis and shown to be elevated in patients with SLE compared with healthy controls $(7,36,37)$. In the present study, using a mouse model, we provide evidence of the essential role of CCL17-producing cells in the progression of end-stage kidney disease, perhaps due to the effect of this chemokine on the recruitment of pathogenic $\mathrm{T}$ cells.

In contrast to the essential role of DCs in end-stage disease, our experiments show that other factors that have been described as mediating lupus nephritis did not necessarily induce a fully destructive pathology: we observed high levels of type I IFN (7, 29), hypoxia (9-11), or changes in iron metabolism in the kidneys (38) in situations in which glomerular function and survival were still maintained. Recognizing the various stages in lupus nephritis and factors that trigger the switch to a more destructive pathology, perhaps with involvement of adaptive responses targeting kidney antigens, will be useful when considering novel therapies specific for patients with the highest severity of lupus nephritis.

\section{Methods}

Experimental animals and infection with malaria parasites. Fc $\gamma$ RIIb-KO (TAC264, Taconic) and C567BL/6J (WT) mice were maintained in specific pathogen-free (SPF) conditions at the NIH. Congenic CD $45.1^{+}$Fc $\gamma$ RIIb-KO mice were generated by crossbreeding
TAC264 mice with JAX mice of the 002014 strain (The Jackson Laboratory). Both female and male mice were used in this study. All efforts were made to minimize animal suffering and to limit the number of animals used.

The rodent malaria parasite used in this work is Plasmodium yoelii 17XNL GFP (23). Either WT or Fc $\gamma$ RIIb-KO were injected i.p. with the amount of blood collected from an infected mouse (3\%-4\% parasitemia) that would contain $10^{6}$ iRBCs. For control mice, the amount of blood equivalent to 1 million infected RBCs was injected. Parasitemia was measured by flow cytometry as the percentage of $\mathrm{GFP}^{+} \mathrm{RBC}$ in the blood. Details of the experimental design are described in Supplemental Figure 1.

ANA analysis. The detection and measurement of ANA levels were done using 12-well microscope slides coated with Hep-2 cells (Hep-2 kit, MBL). Serum dilutions were incubated over the Hep-2 cells for 30 minutes at room temperature (RT). After a PBS wash, Alexa Fluor 488-conjugated anti-mouse IgG was added at $0.83 \mu \mathrm{g} /$ $\mathrm{mL}$ in PBS for 30 minutes at RT. Cells were washed 3 times with PBS and visualized by fluorescence microscopy. Using positive and negative control samples, ANA scores were defined by fluorescence detection in the following serum dilutions: ANA score of 1 (1:100), ANA 2 (1:300), ANA 3 (1:900), ANA 4 (1:2700), and ANA 5 (1:8100). Autoantigen Microarray Super Panel I (UT Southwestern Medical Center) (24) was used to analyze serum IgG and IgM autoantigen specificities. Briefly, after DNAse I treatment, serum samples were diluted 1:50 and incubated using 128-plex autoantigen arrays. For detection, the antibodies were incubated with Cy3-labeled anti-IgG, and the arrays were scanned with a GenePix 4400A Microarray Scanner and analyzed using GenePix 7.0 software.

Proteinuria. Proteinuria was measured using urinalysis dipsticks (Chemstrip 2GP, Thermo Fisher Scientific) and semiquantitatively categorized as $0(<30 \mathrm{mg} / \mathrm{dL}), 1(30 \mathrm{mg} / \mathrm{dL}), 2(100 \mathrm{mg} / \mathrm{dL})$, and 3 (500 mg/dL).

Serum cytokines and antibodies. Serum cytokines were measured using the BD Cytometric Bead Array (CBA) Mouse Inflammation Kit (catalog 552364) following the manufacturer's instructions. Data were acquired in FACSCalibur and LSRII cytometers (BD Biosciences), and the data were analyzed using FCAP software (BD Biosciences). Serum IgG, IgG1, IgG2a/c, IgG2b, IgG3, and IgM levels were measured by ELISA using goat anti-mouse antibodies from SouthernBiotech and an EnSpire Multimode Plate Reader (PerkinElmer). Supplemental Table 1 provides a detailed description of the antibodies used.

Chemokine depletion in vivo was accomplished by injection of anti-CCL17/TARC (MAB529, R\&D Systems), anti-CCL20/MIP-3 $\alpha$ (MAB760, R\&D Systems), or anti-IgG2a isotype control (MAB006, R\&D Systems) antibodies.

Serum transfer assay. Serum was taken from infected or noninfected Fc $\gamma$ RIIb-KO mice 1 month after infection and injected i.v. into 2-month-old Fc $\gamma$ RIIb-KO mice. Serum $(200 \mu \mathrm{L})$ was injected on days 1,7 , and 15. The development of lupus nephritis was analyzed for 7 months by measuring proteinuria.

Tissue preparation and staining. For immunofluorescence staining, fresh kidney tissues were frozen in dry ice using TFM Tissue Freezing Medium (Thermo Fisher Scientific) and maintained at $-80^{\circ} \mathrm{C}$ until use. The $6 \mu \mathrm{m}$ serial cryosections obtained were dried for 1 hour at RT and fixed with $100 \%$ acetone for 10 minutes at $-20^{\circ} \mathrm{C}$. Sections were rehydrated with PBS and blocked with PBS containing 2\% BSA for 1 hour. 
After 3 washes with PBS-Tween 0.05\% and 1 wash with PBS, sections were stained with FITC-conjugated anti-IgG1, anti-IgG2a, anti-IgG2b (all from BD Pharmingen), and anti-complement C3 (MP Biomedicals) antibodies diluted in PBS 1\% BSA for 1 hour at RT. Sections were washed as described above and mounted onto coverslips for image analysis.

For light imaging, tissues were fixed in formalin and embedded in paraffin for sectioning. Sectioned samples were stained with $\mathrm{H} \& \mathrm{E}$, PAMS, and TUNEL. Slides were then incubated with TdT (M0315L, New England Biolabs), and the reaction was detected with antidigoxigenin-peroxidase (Roche Diagnostics) and DAB. Slides were counterstained with hematoxylin and coverslipped for microscopy observation. Also, antibodies including anti-CD45 (5C16, Thermo Fisher Scientific), CD3 (SP7, Abcam), IBA/1 (FUJIFILM Wako Chemicals), arginase 1 (C-2, Santa Cruz Biotechnology), F4/80 (CI:A3-1, Bio-Rad), CD11c (D1V9Y, Cell Signaling Technology), CD45R/B220 (RA3-6B2, BioLegend), and myeloperoxidase (Abcam) were used for the detection of different leukocyte populations (see Supplemental Table 1 for a detailed description of the antibodies used).

Kidney histopathology. H\&E-stained kidney sections were analyzed and scored in a blinded manner by an expert pathologist, who followed the NIH activity and chronicity indexes revisited by the International Society of Nephrology/Renal Pathology Society classification for lupus nephritis (26). We considered the following characteristics in the NIH activity index: endocapillary hypercellularity, neutrophils/karyorrhexis, fibrinoid necrosis, hyaline deposits, cellular/fibrocellular crescents, and interstitial inflammation. For the NIH chronicity index, total glomerulosclerosis score, fibrous crescents, tubular atrophy, and interstitial fibrosis were considered. Histological measurements (number, size, and inflammation of glomeruli, tubular inflammation, and protein deposits) were done from H\&E-stained and PAMS images using ImageJ software (NIH).

Detection of infiltrating cells. Anti-mouse CD45.2 BV421 (3 $\mu \mathrm{g})$ in PBS was injected i.v. into each mouse for vasculature leukocyte discrimination. After 3 minutes, mice were euthanized, and tissues were extracted and processed for flow cytometric analysis. Decapsulated kidney and liver were incubated at $37^{\circ} \mathrm{C}$ with the Multi Tissue Dissociation Kit 1 and dissociated with gentleMACS Octo Dissociator (Miltenyi Biotec) following the manufacturer's instructions. The dissociated solution was washed with PBS 5\% FBS (centrifuged at $300 \mathrm{~g}$ for 5 minutes) and incubated for 5 minutes with ACK Lysis Buffer (Lonza) at RT to eliminate RBCs from the suspension. If necessary, after sample filtration with a $70 \mu \mathrm{M}$ strainer and washing, $\mathrm{CD} 45^{+}$cells were isolated using anti-CD45 magnetic beads and autoMACS Pro Separator (Miltenyi Biotec) at $4^{\circ} \mathrm{C}$.

Flow cytometry staining. Single-cell suspensions obtained from the spleen, LNs, kidneys, and liver were resuspended in staining solution (PBS containing 2\% FBS) and blocked with $0.1 \mathrm{mg} / \mathrm{mL}$ purified rat anti-mouse CD16/CD32 (BD Biosciences) for 15 minutes at $4^{\circ} \mathrm{C}$. Samples were stained with the corresponding conjugated antibodies diluted 1:200 for 30 minutes at $4^{\circ} \mathrm{C}$. If necessary, cells were fixed with PFA 1\% in PBS for 30 minutes, washed, and resuspended in PBS. Live and dead cells were discriminated using the LIVE/DEAD Fixable Yellow Dead Cell Stain Kit (Thermo Fisher scientific). Samples were acquired on an LSR II (BD Biosciences) or CytoFLEX (Beckman Coulter) flow cytometers and then analyzed with FlowJo (Tree Star) or CytExpert (Beckman Coulter) software. The antibodies used are listed in Supplemental Table 1.
BM transfer experiments. BM from Fc $\gamma$ RIIb-KO CD45.1 ${ }^{+}$infected or noninfected mice was transferred 1 month after infection into lethally irradiated 2-month-old Fc $\gamma$ RIIb-KO mice. BM cells (20 million) were extracted from the bone by high-speed centrifugation and reinjected i.v. into recipient mice in $200 \mu \mathrm{L}$ PBS. Recipient mice were irradiated at 9.40 Gy the day before. Reconstitution efficacy was analyzed by flow cytometry 1 month after transfer, measuring the percentage of CD45.1 versus CD 45.2 cells in blood.

qPCR analysis. For gene expression analysis using whole-kidney RNA, fresh tissue was frozen at $-80^{\circ} \mathrm{C}$ in RNAlater solution (Thermo Fisher Scientific) for future use. Samples were dissociated in RLT lysis buffer (QIAGEN) with gentleMACS Octo Dissociator, and the RNA was extracted using the RNeasy Mini Kit (QIAGEN). For gene expression analysis of specific cell populations in the kidney, kidneys were digested at $37^{\circ} \mathrm{C}$ with the Multi Tissue Dissociation Kit 1 and dissociated with gentleMACS Octo Dissociator. Then, the AutoMacs Pro Separator was used to select positive and negative fractions using anti-CD45, anti-CD11b, or anti-CD4 MicroBeads (all from Miltenyi Biotec). In the last case, further purity was achieved by sort purification with TCRb and CD4 antibodies using the FACSAria cell sorter (BD Biosciences).

RNA (0.5-2 $\mu \mathrm{g})$ from samples was transcribed into cDNA, and genomic DNA was eliminated in pretreatment with a $\mathrm{RT}^{2}$ First Strand Kit (QIAGEN). qPCR was performed with $\mathrm{RT}^{2}$ SYBR Green qPCR Mastermix (QIAGEN) and the Cytokines \& Chemokines RT ${ }^{2}$ Profiler PCR Array kit using a CFX Connect Real-Time system and CFX analysis software (Bio-Rad). Data analysis was performed following the $\triangle \Delta \mathrm{Ct}$ method using QIAGEN Data Analysis (https://dataanalysis2. qiagen.com/pcr), which calculates fold change and regulation using the $\Delta \Delta \mathrm{Ct}$ method, in which the $\Delta \mathrm{Ct}$ is calculated between the gene of interest (GOI) and the average of the reference genes (HKG), followed by $\Delta \Delta \mathrm{Ct}$ calculations ( $\Delta \mathrm{Ct}$, test group; $-\Delta \mathrm{Ct}$, control group). The fold change was then calculated using the $2^{\wedge}(-\Delta \Delta \mathrm{Ct})$ formula. Student's $t$ test of the replicate $2^{\wedge}(-\Delta \mathrm{Ct})$ values was performed for each gene in the control group and treatment groups. For kidney cell suspensions, the following primers from Integrated DNA Technologies (IDT) were used: Ccl2O (Mm.PT.58.13906306), Ccl17 (Mm.PT.58.9199490), Actb (Mm.PT.39a.22214843.g), Ifn2 (Mm.PT.58.13701797), Tnf (Mm. PT.58.12575861), Aco1 (Mm.PT.58.5400066), and Hifla (330890334). The primers used are listed in Supplemental Table 2. Data analysis was done using the $\Delta \mathrm{Ct}$ method, normalized to Actb expression.

Statistics. Statistical significance was calculated using GraphPad Prism 7.0 (GraphPad Software). For comparisons between 2 normally distributed groups, a 2-tailed, unpaired $t$ test was used. Nonparametric data were analyzed using the Mann-Whitney $U$ test. For comparisons between more than 2 groups, statistical analysis was performed using ANOVA for parametric data and the Kruskal-Wallis test for nonparametric data. For correlation analysis, Pearson's or Spearman's coefficients were used for parametric or nonparametric data. Differences in the mortality rates of mice were assessed by Cox-Mantel log-rank test. Data indicate the mean \pm SD. A $P$ value of less than 0.05 was considered statistically significant.

Study approval. This research was conducted in accordance with the NIAID's Animal Care and Use Committee (ACUC) policies and procedures. All animals were housed and studied under approved NIH ACUC guidelines. All experiments were approved by the ACUC of the NIAID, NIH (project name: LIG1OE). 


\section{Author contributions}

LA conceptualized the study, performed experiments, and wrote the original draft of the manuscript. BS provided mouse technical support. HKK and JW performed experiments. CFQ performed pathological review. SB supervised the study and wrote, reviewed, and edited the manuscript.

\section{Acknowledgments}

We are grateful to Louis Miller for helpful discussions, and Susan Pierce, Brian Dixon, and Hongsheng Wang for help with editing the manuscript. We thank Susan Pierce's group for providing the
P. yoelli parasite. We thank the staff of Twinbrook II Charles River Laboratories for animal husbandry. This research was supported by the Intramural Research Program of the NIH, NIAID (project no. ZIA AI000912).

Address correspondence to: Silvia Bolland, NIAID, NIH, 5625 Fishers Lane 4N03a, Rockville, Maryland 20814, USA. Phone: 301.443.3158; Email: sbolland@nih.gov.

JW's present address is: Department of Nephrology, Zhejiang Provincial People's Hospital, Zhejiang 310014, China.
1. Anders HJ, et al. Lupus nephritis. Nat Rev Dis Primers. 2020;6(1):7.

2. Maria NI, Davidson A. Protecting the kidney in systemic lupus erythematosus: from diagnosis to therapy. Nat Rev Rheumatol. 2020;16(5):255-267.

3. Daniel JW, Hahn B, eds. Dubois' Lupus Erythematosus and Related Syndromes. Elsevier; 2018.

4. Pisetsky DS, Lipsky PE. New insights into the role of antinuclear antibodies in systemic lupus erythematosus. Nat Rev Rheumatol.2020;16(10):565-579.

5. Nowling TK, Gilkeson GS. Mechanisms of tissue injury in lupus nephritis. Arthritis Res Ther. 2011;13(6):250.

6. Yu F, et al. Redefining lupus nephritis: clinical implications of pathophysiologic subtypes. Nat Rev Nephrol. 2017;13(8):483-495.

7. Der E, et al. Tubular cell and keratinocyte single-cell transcriptomics applied to lupus nephritis reveal type I IFN and fibrosis relevant pathways. Nat Immunol. 2019;20(7):915-927.

8. Bethunaickan R, et al. Identification of stage-specific genes associated with lupus nephritis and response to remission induction in $(\mathrm{NZB} \times \mathrm{NZW})$ F1 and NZM2410 mice. Arthritis Rheumatol. 2014;66(8):2246-2258.

9. Deng $\mathrm{W}$, et al. Hypoxia inducible factor-1 alpha promotes mesangial cell proliferation in lupus nephritis. Am J Nephrol. 2014;40(6):507-515.

10. Kurata $Y$, et al. The role of hypoxia in the pathogenesis of lupus nephritis. Kidney Int. 2020;98(4):821-823.

11. Chen PM, et al. Kidney tissue hypoxia dictates $\mathrm{T}$ cell-mediated injury in murine lupus nephritis. Sci Transl Med.2020;12(538):eaay1620.

12. Kang $S$, et al. BAFF induces tertiary lymphoid structures and positions $\mathrm{T}$ cells within the glomeruli during lupus nephritis. J Immunol. 2017;198(7):2602-2611.

13. Teichmann LL, et al. Dendritic cells in lupus are not required for activation of $\mathrm{T}$ and $\mathrm{B}$ cells but promote their expansion, resulting in tissue damage. Immunity. 2010;33(6):967-978

14. Bethunaickan $\mathrm{R}$, et al. A unique hybrid renal mononuclear phagocyte activation phenotype in murine systemic lupus erythematosus nephritis. JImmunol. 2011;186(8):4994-5003.

15. Schiffer $\mathrm{L}$, et al. Activated renal macrophages are markers of disease onset and disease remission in lupus nephritis. JImmunol.2008;180(3):1938-1947.

16. Heymann F, et al. Kidney dendritic cell activation is required for progression of renal disease in a mouse model of glomerular injury. JClin Invest. 2009;119(5):1286-1297.

17. Kim AH, et al. New roles revealed for T cells and DCs in glomerulonephritis. JClin Invest. 2009;119(5):1074-1076.

18. Bosteels C, et al. Inflammatory type $2 \mathrm{cDCs}$ acquire features of $\mathrm{CDC} 1 \mathrm{~s}$ and macrophages to orchestrate immunity to respiratory virus infection. Immunity. 2020;52(6):1039-1056.

19. Kurts C, et al. Kidney dendritic cells: fundamental biology and functional roles in health and disease. Nat Rev Nephrol. 2020;16(7):391-407.

20. Greenwood BM, et al. Suppression of autoimmune disease in NZB and (NZB x NZW) F1 hybrid mice by infection with malaria. Nature. 1970;226(5242):266-267.

21. Butcher G. Autoimmunity and malaria. Trends Parasitol. 2008;24(7):291-292.

22. Bolland S, Ravetch JV. Spontaneous autoimmune disease in $\mathrm{Fc}$ (gamma)RIIB-deficient mice results from strain-specific epistasis. Immunity. 2000;13(2):277-285.

23. Ono T, et al. Plasmodium yoelii yoelii 17XNL constitutively expressing GFP throughout the life cycle. Exp Parasitol. 2007;115(3):310-313.

24. Zhu H, et al. Autoantigen microarray for high-throughput autoantibody profiling in systemic lupus erythematosus. Genomics Proteomics Bioinformatics. 2015;13(4):210-218.

25. Hentati B, et al. Beneficial effect of polyclonal immunoglobulins from malaria-infected BAL$\mathrm{B} / \mathrm{c}$ mice on the lupus-like syndrome of (NZB $\mathrm{x}$ NZW)F1 mice. Eur J Immunol. 1994;24(1):8-15.

26. Bajema IM, et al. Revision of the International Society of Nephrology/Renal Pathology Society classification for lupus nephritis: clarification of definitions, and modified National Institutes of Health activity and chronicity indices. Kidney Int.
2018;93(4):789-796.

27. Anderson SA, et al. The IRP1-HIF-2alpha axis coordinates iron and oxygen sensing with erythropoiesis and iron absorption. Cell Metab 2013;17(2):282-290.

28. Hallan S, et al. Metabolomics and gene expression analysis reveal down-regulation of the citric acid (TCA) cycle in non-diabetic CKD patients. EBioMedicine. 2017;26:68-77.

29. Fairhurst AM, et al. Type I interferons produced by resident renal cells may promote end-organ disease in autoantibody-mediated glomerulonephritis. J Immunol. 2009;183(10):6831-6838.

30. Alferink J, et al. Compartmentalized production of CCL17 in vivo: strong inducibility in peripheral dendritic cells contrasts selective absence from the spleen. JExp Med. 2003;197(5):585-599.

31. Greenwood BM, et al. Speckled antinuclear factor in African sera. Clin Exp Immunol. 1970;7(1):75-83.

32. Adu D, et al. Anti-ssDNA and antinuclear antibodies in human malaria. Clin Exp Immunol. 1982;49(2):310-316.

33. Greenwood BM, et al. Can parasitic infection suppress autoimmune disease? Proc R Soc Med. 1970;63(1):19-20

34. Badr G, et al. Infection of female BWF1 lupus mice with malaria parasite attenuates $B$ cell autoreactivity by modulating the CXCL12/CXCR4 axis and its downstream signals PI3K/AKT, NFKB and ERK. PLoS One. 2015;10(4):e0125340.

35. Imai $\mathrm{T}$, et al. Involvement of $\mathrm{CD} 8^{+} \mathrm{T}$ cells in protective immunity against murine blood-stage infection with Plasmodium yoelii 17XL strain. Eur J Immunol. 2010;40(4):1053-1061.

36. Okamoto H, et al. A role for TARC/CCL17, a CC chemokine, in systemic lupus erythematosus. J Rheumatol. 2003;30(11):2369-2373.

37. Stanley S, et al. Identification of low-abundance urinary biomarkers in lupus nephritis using electrochemiluminescence immunoassays. Arthritis Rheumatol. 2019;71(5):744-755.

38. Marks ES, et al. Renal iron accumulation occurs in lupus nephritis and iron chelation delays the onset of albuminuria. Sci Rep. 2017;7(1):12821. 